
\title{
3 Research Suare \\ LINC00893 Inhibits The Progression of Prostate Cancer Through miR-3173-5p/SOCS3/JAK2/STAT3 Pathway
}

\section{Chuigong Yu}

General Hospital of People's Liberation Army: Chinese PLA General Hospital

\section{Yu Fan}

General Hospital of People's Liberation Army: Chinese PLA General Hospital

\section{Yu Zhang}

General Hospital of People's Liberation Army: Chinese PLA General Hospital

Lupeng Liu

General Hospital of People's Liberation Army: Chinese PLA General Hospital

Gang Guo ( $\nabla$ weiu116@163.com )

General Hospital of People's Liberation Army: Chinese PLA General Hospital https://orcid.org/00000003-3378-3657

\section{Primary research}

Keywords: LINC00893, prostate cancer, miR-3173-5p, SOCS3, JAK2/STAT3 signaling pathway

Posted Date: September 9th, 2021

DOI: https://doi.org/10.21203/rs.3.rs-809513/v1

License: (c) (i) This work is licensed under a Creative Commons Attribution 4.0 International License. Read Full License 


\section{Abstract}

Background: Prostate cancer $(\mathrm{PCa})$ is one of the most common malignant tumors in the male urinary system. In recent years, the morbidity and mortality of PCa have been increasing due to the limited effects of existing treatment strategies. Long non-coding RNA (IncRNA) LINC00893 inhibits the proliferation and metastasis of papillary thyroid cancer (PTC) cells, but its role in PCa has not been reported. Our study aims to clarify the role and underlying mechanism of LINC00893 in regulating the progression of PCa.

Methods: We analyzed LINC00893 expression through TCGA database. We also collected 66 paires of PCa tissues and matched para-cancerous tissues as well as cell lines and assessed LINC00893 expression. Subsequently, we conducted gain-of-function assays to confirm the role of LINC00893 in PCa. CCK-8, EdU, colony information and transwell assays were implemented to detect cell proliferation, colony formation and metastasis abilities, respectively. RT-qPCR and western blot assays were used to quantify the expression of mRNA and protein. Dual-luciferase reporter, RNA-binding protein immunoprecipitation (RIP) and RNA pull down assays were conducted to evaluate the interaction of molecules. Spearman correlation coefficient analysis was conducted to detect the correlation between molecules.

Results: We found that the LINC00893 expression in PCa tissues and cell lines was upregulated compared with matched controls, and patients with low expression of LINC00893 suffered a low overall survival rate. Overexpression of LINC00893 hindered the proliferation, epithelial-mesenchymal transition (EMT) as well as metastasis of PCa cells in vitro and in vivo. In terms of mechanism, suppressor of cytokine signaling 3 (SOCS3)/Janus Kinase 2 (JAK2)/signal transducer and activator of transcription 3 (STAT3) signaling pathway occupied a central position in the regulation of PCa progression by LINC00893. LINC00893 weakened the inhibition role of miR-3173-5p on SOCS3 expression through functioning as a miR-3173-5p sponge, which inhibited the JAK2/STAT3 signaling pathway.

Conclusions: LINC00893 suppresses the progression of prostate cancer through miR-31735p/SOCS3/JAK2/STAT3 pathway. our data uncovers a novel mechanism by which LINC00893 hinders the progression of $\mathrm{PCa}$, which enriches the molecular network of LINC00893 regulating the $\mathrm{PCa}$ progression and laies a theoretical foundation for PCa targeted therapy.

\section{Introduction}

Prostate cancer (PCa) is the most common epithelial malignant tumor of the male urinary system [1, 2]. Advanced age, heredity, obesity and environmental pollution are the crucial factors leading to PCa [3-6]. At present, the main therapeutic strategies for $\mathrm{PCa}$ include surgical resection, androgen deprivation therapy, radiation therapy as well as chemotherapy [7]. However, the curative effect is always unsatisfactory. Therefore, exploring the molecular mechanism of the pathogenesis and progression of 
PCa, finding novel and effective molecular targets, and implementing precise targeted therapy is the future development trend.

Non-coding RNAs (ncRNAs) exist in most organisms, they do not encode proteins and implicate in cancer cells growth and metastasis. NcRNAs can be assigned to long ncRNAs (IncRNAs) (>200 nt) and short ncRNAs (sncRNA) (<30 nt) according to the transcript length. SncRNA contains microRNAs (miRNAs) and short interfering RNAs (siRNAs) [8-13]. IncRNAs and microRNAs are implicated in the initiation and progression of various cancers including prostate cancer[14], glioma[15] and breast cancer[16], and are potential biomarkers and therapeutic targets $[17,18]$. Accumulating evidences prove that IncRNAs alleviate the inhibitory effect of miRNAs on downstream target mRNAs by functioning as competing endogenous RNAs (ceRNAs) [19]. For example, LncRNA NEAT1 serves as sponges of miR-34a-5p as well as miR-204-5p, thereby enhancing the docetaxel resistance of PCa cells [20]. LncRNA CASC15 targets miR-200a-3p and enhances the metastasis ability of cancer cells in PCa [21]. LncRNA AATBC accelerates the $\mathrm{PCa}$ progression via regulating the miR-1245b-5p/CASK axis [22]. Whereas, the functional role and regulatory mechanism of LINC00893 regulating PCa progression need to be further investigated.

The non-receptor tyrosine kinase Janus kinase 2 (JAK2) /Transducer and Activator of Transcription 3 (STAT3) signaling pathway is stimulated by cytokines $[23,24]$. When the cytokine binds to the receptor on the cell membrane, JAK2 is phosphorylated, and transcription factor STAT3 is further phosphorylated by JAK2 and enter the nucleus, followed by the regulation of STAT3 on a spectrum of downstream target genes, thereby regulating a wide spectrum of biological processes, such as proliferation, differentiation and metastasis of cells[25-27]. The activity of JAK2/STAT3 signaling pathway is hindered by the suppressor of cytokine signaling (SOCS) family. The SOCS family contains SOCS 1-7 as well as cytokine-inducible SH2-containing protein (CIS) $[28,29]$. SOCS3 belongs to SOCS family, which has a higher affinity for the JAK2 compared with SOCS1. SOCS3 guides the degradation of JAK2 by the proteasome or changes the conformation of STAT3, thereby hindering the binding of STAT3 to the gene promoters. Therefore, SOCS3 inhibits the activity of JAK2/STAT3 signaling pathway as the most critical negative regulator [30, 31] [32-34]. Recent studies have illustrated that the SOCS3/JAK2/STAT3 signal axis is implicated in the progression of a spectrum of cancers. For instance, the SOCS3/JAK2/STAT3 signal axis mediates the inhibitory role of piperlongumine in the osteosarcoma progression [35]. The SOCS3/JAK2/STAT3 signaling pathway is as well implicated in hepatocellular carcinoma malignant progression [36]. Furthermore, the SOCS3/JAK2/STAT3 signal axis is closely associated with epithelialmesenchymal transition (EMT) and metastatic ability of gallbladder cancer cells [37]. However, the regulation of SOCS3/JAK2/STAT3 signaling axis in PCa has not been studied yet.

In our study, we compared the differences of LINC00893 expression between PCa tissues and matched para-cancerous tissues through the TCGA database. We further verified the analyzed result in 66 pairs of PCa cancer tissues and PCa cell lines as well as their matched controls, and the data illustrated that LINC00893 was significantly down-regulated in PCa tissues and cell lines. Furthermore, our survival analysis data exhibited that patients with low expression of LINC00893 suffered a lower overall survival rate. The above results laid the foundation for LINC00893 as a anti-tumorigenic factor. Subsequently, we 
performed a gain-of-function assay through overexpressing LINC00893, which showed that LINC00893 inhibited the proliferation, migration, invasion as well as EMT of PCa cells in vitro and in vivo. After that, we explored the underlying mechanism of LINC00893 regulaing PCa progression, and clarified that LINC00893 regulated the malignant phenotype of PCa through the miR-3173-5p/SOCS3/JAK2/STAT3 axis. LINC00893 absorbed miR-3173-5p through sponge effect, which weakened the inhibitory role of miR-3173-5p in SOCS3 and impaired the JAK2/STAT3 signaling pathway activity. In conclusion, our study uncovered a novel mechanism for LINC00893 to regulate the malignant progression of PCa, which may provide a theoretical basis for PCa therapy.

\section{Materials And Methods}

\section{Tissue samples}

A total of 66 paris of PCa tissues and matched para-cancerous tissues were obtained from Department of Urology, The Third Medical Center of Chinese People's Liberation Army General Hospital from February 2018 to September 2020. These patients (Gender: 66 males, Age: 65 to 82 years old) who provided the tissues were diagnosed with PCa and were pathologically confirmed. All patients had not undergone radiotherapy and chemotherapy before $\mathrm{PCa}$ surgical resection, and these patients had complete medical records since admission. Patients with other organic diseases including hypertension, diabetes, metabolic diseases, immune diseases and other tumors were not included in the study. The details of this study have been informed to the patients and the informed consent form signed by them has been obtained. This study has been approved by the Medical Ethics Committee of The Third Medical Center of Chinese People's Liberation Army General Hospital from the collection of samples to the conduction of all research procedures.

\section{Cell culture and transfection}

All PCa cells (PC-3, DU145, VCaP and LNCaP) as well as human normal prostate epithelial cell (RWPE-1) were obtained from American Type Culture Collection (ATCC, Rockville, MD, USA). PC-3, DU145 and RWPE-1 cells were grown in F-12 K basic medium (Invitrogen, California, USA), Eagle's Minimum Essential medium (EMEM, Gibco, California, USA) and Keratinocyte serum free medium (Invitrogen), respectively. $\mathrm{VCaP}$ and LNCaP cells were both grown in RPMI 1640 basic medium (Gibco). All cells culture medium contained $10 \%$ fetal bovine serum (FBS, Gibco) as well as $100 \mathrm{U} / \mathrm{mL}$ penicillin and $100 \mu \mathrm{g} / \mathrm{mL}$ streptomycin (Gibco) except for RWPE-1. All cells were grown in a humidified cell culture incubator under conditions of $37^{\circ} \mathrm{C}$ with $5 \% \mathrm{CO} 2$.

The LINC00893 sequence was cloned into the plasmid expression vector pcDNA3.1 to constructed LINC00893 overexpression plasmid (pcDNA3.1-LINC00893). pcDNA3.1-LINC00893 and pcDNA3.1-vector plasmids were purchased from Genechem Co., Ltd (Shanghai, China). MiR-3173-5p-mimic/inhibtor and siRNA targeting SOCS3 (si-SOCS3) and their matched controls were prepared from RiboBio Co., Ltd. (Guangzhou, China). pcDNA3.1-LINC00893 overexpression plasmids, miR-3173-5p-mimic, miR-3173-5pinhibtor, si-SOCS3 or their matched controls were transfected into cells alone or in combination 
accompanied with the lipofectamine 2000 reagent (Invitrogen) based on recommended methord and dosage of plasmids and siRNAs. The sequences information was as follows: miR-3173-5p-mimic: 5》UGCCCUGCCUGUUUUCUCCUUU-3®, miR-3173-5p-inhibtor: 5囚-AAAGGAGAAAACAGGCAGGGCA-3囚; PC-3 cells were infected with recombinant lentivirus carrying sequence LINC00893 (PC-3- LINC00893) or matched control (PC-3-vector) (Shanghai Genechem Co., Ltd., China). $48 \mathrm{~h}$ post infection, cells stably overexpressing LINC00893 were selected using $1.0 \mu \mathrm{g} / \mathrm{mL}$ puromycin .

\section{Real-time quantitative PCR analysis (RT-qPCR)}

The total RNA in tissues and cells was extacted using trizol method according to the standard method previously described [38]. For the RNA extraction in tissues, $100 \mathrm{mg}$ fresh tissue blocks were fully ground in liquid nitrogen before adding Trizol. The RNA concentration was measured using NanoDorp.

Subsequently, the reverse-transcription of $5 \mu \mathrm{g}$ RNA to CDNA was performed using CDNA Reverse Transcription Kit (Invitrogen, K1691). SYBR premix EX TAQ II kit (Takara, Dalian, China) was used to further amplify cDNA on 7500 Real Time PCR Systerm (Applied Biosystems/Life Technologies, Carlsbad, CA, USA) according to the requirements of the instructions. Lastly, the results were calculated according to $2^{-\Delta \Delta C t}$ method. The relative expression level of genes were normalized to Glyceraldehyde-3-phosphate dehydrogenase (GAPDH). Three independent assays were performed in the RT-qPCR assay in the same method. The primers were synthesized by Sangon Biotechnology Co., Ltd. (Shanghai, China) and were listed below: LINC00893 (Forward: 5囚-AGTCGGCTCACCAATTCGT-3》, Reverse: 5》-

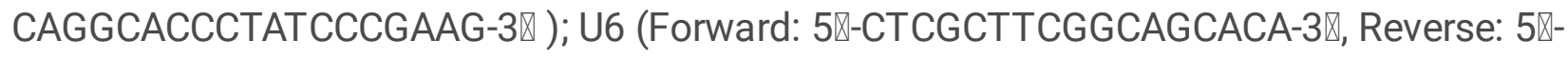
AACGCTTCACGAA TTTGCGT-3区); GAPDH (Forward: 5'-GACAGTCAGCCGCATCTTCT-3', Reverse: 5'GCGCCCAATACGACCAAATC-3'); miR-3173-5p (Forward: 5囚-TGCCCTGCCT GTTTTCTCC-3囚, Reverse: 5'GAACATGTCTGCGTATCTC-3区) ) SOCS3 (Forward: 5囚-CCTGCGCCTCAA GACCTTC-3囚, Reverse: $5 \llbracket$ GTCACTGCGCTCCAG TAGA A-3囚)

\section{CCK-8 cell proliferation assay}

PCa cells with indicated treatment were seeded in 96-well-plates (2000 cell/well). Cells were cultured under conditions of $37^{\circ} \mathrm{C}$ with $5 \% \mathrm{CO} 2$ for $0 \mathrm{~h}, 24 \mathrm{~h}, 48 \mathrm{~h}, 72 \mathrm{~h}$ and $96 \mathrm{~h}$, respectively. Subsequently, 10 $\mu \mathrm{L}$ CCK-8 reaction reagent was added into the cell plates in the indicated time point and incubating for 4 h. Finally, the absorbance at $450 \mathrm{~nm}$ in each condition was captured by a microplate reader (Bio-Rad, Hercules, USA) [39]. CCK-8 cell proliferation assay was conducted with three independent assays in the same method.

\section{5-ethynyl-2-deoxyuridine (EdU) incorporation assay}

EdU incorporation was detcted employing the EdU assay kit (C10310-1, RiboBio, Guangzhou, China) according to the requirements of the instructions. Briefly, PCa cells PC-3 and LNCaP were seeded to 24well-plates ( 1 i $10^{5}$ cells/well) and cultured overnight. Then, cells were transfected with pcDNA3.1LINC00893 overexpression plasmids or pcDNA3.1-vector using lipofectamine 2000 (Invitrogen). 48h post transfection, cells were incubated with $200 \mu \mathrm{L} 50 \mu \mathrm{m}$ EdU for $2 \mathrm{~h}$, followed by fixing with $2 \%$ 
paraformaldehyde (PFA) for $20 \mathrm{~min}$ and $2 \mathrm{mg} / \mathrm{mL}$ glycine was employed to neutralize PFA. After that, cell membrane was destroyed employing $0.1 \%$ Triton X-100 for $10 \mathrm{~min}$. Lastly, cells were stained with $100 \mu \mathrm{L}$ Apollo staining solution for 30 mins and with 4',6- diamidino-2-phenylindole (DAPI) DNA staining solution for 20 mins. The Fluorescence images were collected and EdU-positive cells were counted usingr a microscope (Nikon, Japan)[40]. Three independent assays was performed in the EdU assay in the same method.

\section{Colony formation assay}

PC-3 and LNCaP cells treated as indication were resuspended in fresh medium and then seeded into the 6-well-plates (1000 cells/well). Then, cells were grown under conditions of $37^{\circ} \mathrm{C}$ and $5 \% \mathrm{CO} 2$ for 14 days. Subsequently, the culture medium was discarded and $4 \%$ paraformaldehyde was employed to fix the cells for 20 mins. After that, cells were colored using Giemsa Stain Kit (Abcam, ab150670) for 20 mins. Lasly, images of typical colony morphology were photographed and the the number of colonies in each conditions was counted under a Leica AM6000 microscope [41]. The colony formation assay was conducted with three independent assays in the same method.

\section{Cell migration and invasion assay}

Cell metastesis ability was assessed using teanswell experiment as the method in previous study[42]. PC3 and LNCaP cells were seeded into 6-well-plates, followed by the indicated transfection. 48h post transfection, cells were resuspended in serum-free culture medium. For invasion, the 24-well transwell upper chamber (Sigma, Germany) was pre-coated with $500 \mathrm{ng} / \mathrm{ml}$ Matrigel (BD Biosciences, Bedford, MA), while For migration, the transwell upper chamber was not pre-coated by Matrigel. A total of 1 i $10^{4}$ resuspended cells were seeded into the upper chamber with or without Matrigel in serumfree culture medium. $500 \mu \mathrm{L}$ fresh culture medium with $10 \%$ FBS was added in the lower chamber. Subsequently, cells were cultured for $48 \mathrm{~h}$. $48 \mathrm{~h}$ later, The cells in the upper chamber were wiped off by swabs, followed by fixation with $4 \%$ paraformaldehyde for 10 mins and staining with $0.5 \%$ crystal violet solution for 20 mins. Lastly, migration and invasion cells were counted and typical images were collected under a Leica DC 300F microscope. The cell migration and invasion assays were carried out in three independent assays. Six visual fields were randomly selected and counted in the per chamber of each assay, then the mean of the six visual fields was calculated and the data of three independent assays were used for making figures.

\section{Western blot (WB)}

The abundance of proteins was evaluated using western blot based on the method in previous study[42]. The proteins in cells with indicated treatment were extracted using RIPA Lysis Buffer. Lysis Buffer containing proteins were centrifuged at a speed of $12000 \mathrm{rpm}$ to remove cell debris. Then, Protein concentration was detected by BCA kit (Thermo Fisher Scientific, Waltham, MA, USA) and 5îloading buffer was added into the resulted Lysis Buffer at a ratio of 1: 4, followed by denaturation of proteins at $100^{\circ} \mathrm{C}$ for 10 mins. $40 \mu \mathrm{g}$ of prepared protein was separated in SDS-PAGE systerm and further transfered 
to polyvinylidene fluoride (PVDF) membrane (BioRed, USA) for $2 \mathrm{~h}$. Subsequently, the protein-carrying PVDF membrane was blocked in $5 \%$ skimmed milk for $1 \mathrm{~h}$ at room temperature. After that, protein-carrying PVDF membrane was incubated with primary antibodies anti-E-Cadherin (CST, \#3195, 1:1000), anti-NCadherin (CST, \#14215, 1:1000), anti-vimentin (CST, \#5741, 1:1000), anti-GAPDH (CST, \#2118, 1:2000), anti-SOCS3 (CST, \#52113, 1:1000) , anti-Phospho-JAK2 (CST, \#3771, 1:1000), anti-JAK2 (CST, \#74987, 1:1000) anti-p-STAT3 (CST, \#9145, 1:1000) and anti-STAT3 (CST, \#12640, 1:1000) at $4^{\circ} \mathrm{C}$ overnight and then washed 3 times with 1ÎTBST buffer (5 mins each time). Subsequently, the protein-carrying PVDF membrane was incubated with HRP-labeled secondary antibody (Anti-rabbit lgG, HRP-linked Antibody: CST, \#7074, 1:2000; Anti-mouse IgG, HRP-linked Antibody: CST, \#7076, 1:2000) for $1 \mathrm{~h}$ at room temperature and then washed 5 times (10 mins each time). Finally, the protein bands were developed employing enhanced chemiluminescence reagent (ECL, Thermo Fisher Scientific) and the ralative protein abundance was quantified employing ImageJ software. The western blot was conducted in three independent assays in the same method.

\section{RNA Subcellular Isolation}

The RNAs accumulating in cell cytoplasm and nucleus were seperated using RNA Subcellular Isolation Kit (25501, Active Motif, California, USA.). Briefly, cells growing in $150 \mathrm{~mm}$ plate at $90 \%$ confluence were harvested with trypsin followed by centrifugation at $14000 \mathrm{rpm}$ for $5 \mathrm{~min}$ and the supernatant was disgarded. Subsequently, each cell pellet was resuspended in $120 \mu \mathrm{L}$ complete lysis buffer and incubated for 10 mins on ice. After that, cell sample was centrifuged at $14000 \mathrm{rpm}$ for $5 \mathrm{mins}$. Finally, the cytoplasm RNAs were extracted from the supernatant and the nucleus RNAs were extracted from the pellet. The resulted RNA was dissolved in DEPC water. The RNA quantification and reverse-transcription as well as the detection of expression levels of U6 small nuclear RNA (U6 snRNA), GAPDH and LINC00893 were performed based on the method described in RT-qPCR analysis. U6 snRNA and GAPDH were used as the markers of nucleus and cytoplasm, respectively. Three independent assays were performed in the same method for RNA Subcellular Isolation assay

\section{Dual-luciferase reporter assay}

Dual-Luciferase reporter assay was conducted according to the method in the previous study [43]. The binding sites between LINC00893 and miR-3173-5p, or miR-3173-5p and SOCS3 were analyzed through Starbase database. LINC00893 sequences with the wide-type (WT) or Mutant (MUT) binding site between LINC00893 and miR-3173-5p were cloned into the pmirGLO luciferase reporter plasmid vector (Promega, Madison, WI, USA) to construct WT (pmirGLO-WT-LINC00893) and MUT (pmirGLO-MUTLINC00893) luciferase reporter plasmid. The WT/MUT luciferase reporter plasmid and miR-NC/ miR3173-5p and Renilla luciferase (Rluc) control plasmids were co-transfected into PC-3 and LNCaP cells using lipofectamine 2000 (Invitrogen). The detection of miR-3173-5p binding to SOCS3 was similar to the above method. The SOCS3 mRNA sequence containing the WT or MUT binding site of miR-3173-5p and SOCS3 was inserted into the pmirGLO luciferase reporter plasmid vector (Promega) to product the WT (pmirGLO-WT-SOCS3) and MUT (pmirGLO-MUT-SOCS3) luciferase reporter plasmid. Subsequently, the 
luciferase reporter plasmid and miR-NC/miR-3173-5p as well as Renilla luciferase (Rluc) control plasmids were co-transfected into cells. $48 \mathrm{~h}$ post co-transfection, the relative luciferase activity was assessed depending on the Dual-Luciferase ${ }^{\circledR}$ Reporter Assay System (Promega, E1910) using a luminescence microplate reader (Infinite 200 PRO; Tecan). Renilla luciferase (hRlucneo) control plasmid was used as a internal reference. The dual-luciferase reporter assay was conducted with three independent assays in same method.

\section{RNA-binding protein immunoprecipitation (RIP) assay}

RIP assay was conducted to detect according to the method reported in previous study[44]. The functional interaction between LINC00893 and miR-3173-5p was assessed employing Magna RIP ${ }^{\text {TM }}$ RNABinding Protein Immunoprecipitation Kit ( Millipore, Billerica, MA, USA) according to the manufacturer's instructions. Briefly, PC-3 and LNCaP cells were lysed with RIP lysis bufer pre-added with RNasin (Takara) as well as protease inhibitor cocktail (Thermo Fisher Scientific). Then, resulted cell lysate was centrifugated at $12000 \mathrm{rpm}$ for 30 mins to remove cell debris. Subsequently, magnetic beads conjugated with anti-IgG (Millipore) or anti-Argonaute 2 (Ago2) antibodies (Millipore)were added to the resulted cell lysate and incubated overnight at $4^{\circ} \mathrm{C}$, followed by washing twice with washing buffer and incubation with proteinase K. Lastly, RNA was extracted and the quantified of LINC00893 and miR-3173-5p was performed using RT-qPCR method. Three independent assays were carried out in same method for RIP assay.

\section{RNA pull down assay}

Cells were transfected with $50 \mathrm{nM}$ miR-3173-5p or miR-NC conjugated with biotin for $48 \mathrm{~h}$. Then, cells were lysed by cell lysis buffer (Ambion, Austin, TX, USA), followed by the centrifugation at a speed of $14000 \mathrm{rpm}$ for $10 \mathrm{~min}$. Then, $20 \mu \mathrm{L}$ supernatant was take out as the input group, and the remaining supernatant was incubated with M-280 streptavidin magnetic beads (Sigma) pre-blocked by yeast tRNA and RNase-free BSA (Sigma) at $4^{\circ} \mathrm{C}$ overnight. Lastly, beads-Bio-RNA complex was rinsed and the RNA was extracted using trizol method. The interation between LINC00893 and miR-3173-5p was evaluated employing RT-qPCR[45]. Three independent assays were conducted in the same method for RNA pull down assay.

\section{Xenograft tumorigenesis assay}

A total of 1 I $10^{6}$ PC-3 cells stably overexpressing LINC00893 (PC-3-LINC00893) or its matched control cells (PC-3-vector) were subcutaneously inoculated into the left armpit of 6-week-old male NOD/SCID mice ( $n=6$, Gempharmatech. Co., Ltd,). The tumor volume was detected every 5 days. Tumor-bearing mice were sacrificed using pentobarbital solution $(150 \mathrm{mg} / \mathrm{kg}$, i.p.) without pain on the 30 th day, and the subcutaneous tumors were removed and weighed. Subsequently, the tumor tissues were used for immunohistochemistry $(\mathrm{IHC})$ staining and the RNA and protein in tumor tissues were extracted. The animal study was approved by the The Laboratory Animal Ethics Committee of The Third Medical Center of Chinese People's Liberation Army General Hospital. 


\section{Immunohistochemistry}

Xenograft mice tumor tissues were first fixed with $4 \%$ paraformaldehyde and then embedded in paraffin. The paraffin-embedded tissues were cut into $5 \mu \mathrm{m}$ tissue sections and fixed on glass slides. Paraffinembedded tissue sections were baked at $65^{\circ} \mathrm{C}$ for $2 \mathrm{~h}$ and then submerged in xylene to deparaffinized followed by hydration with gradient alcohol (100\%-100\%-95\%-90\%-80\%-70\%). Subsequently, pH 6.0 citrate buffer was added to the pressure cooker and then the tissue sections were submerged in it for antigen retrieval. After that, $1 \% \mathrm{H} 2 \mathrm{O} 2$ was added to tissue sections and incubated for 20 mins. Then, the tissues were incubated with primary antibodies anti-Ki67 (CST, \#9027, 1: 200), anti-SOCS3 (Santa, \# sc-518020, 1:200) overnight at $4^{\circ} \mathrm{C}$, followed by the washing with 1îPBS for 3 times. Then the tissues were incubated with secondary antibody (GE Healthcare, Piscataway, NJ, USA) for $1 \mathrm{~h}$ at room temperature. The color was developed using DAB kit (Maxin, Fuzhou, China) and the staining indensity was observed employing a microscope. After that, The tissue sections were counterstained by hematoxylin for $1 \mathrm{~min}$, were dehydrated, and then were sealed with neutral resin. Finally, color intensity was analyzed using a Leica DC 300F microscope.

\section{Statistical analysis}

Data statistical analysis was conducted using SPSS 13 software and Graphpad Prism 6.0 software was employed to product the figures. The data was presented in the form of mean \pm standard deviation (SD). For the Statistical method, The comparison of the difference between the experimental group and the control group was conducted by student's t-test. The comparison of the difference among multiple groups (more than two groups) was performed using one-way analysis of variance (ANOVA). The difference in survival time of patients in LINC00893-low and LINC00893-high groups was compared adopting log-rank test. Two-way ANOVA was used to compare the differences in cell proliferation capacity in vitro and tumor volume in vivo under different treatment conditions at different time points. Correlation analysis was performed to compare the correlation between the expression levels of the two molecules. Generally, $\mathrm{P}<0.05$ was considered statistically significant.

\section{Results}

\section{LINC00893 is low expressed in PCa tissues and cell lines}

To seek clinical support, we analyzed the LINC00893 expression in PCa tissues depended on the GSE73397_GSE26910 datasets derived from the TCGA database. The analysis showed that the LINC00893 expression in PCa tissues was reduced compared with matched para-cancerous tissues (Fig. 1A). Subsequently, we collected 66 pairs of PCa tissues as well as matched adjacent normal tissues, and identified that the LINC00893 expression in PCa tissues was reduced compared with that of matched para-cancerous tissues (Fig. 1B). Furthermore, the LINC00893 expression in PCa cell lines PC-3, DU145, VCaP and LNCaP was also lower compared with human normal prostate epithelial cell line RWPE-1 (Fig. 1C). To further explore the ralationship between LINC00893 and survival of PCa patients. We assigned 66

PCa patients to LINC00893 low expression group $(n=33)$ and LINC00893 high expression group $(n=33)$ 
depended on the median value of LINC00893 expression as the cut-off value in Fig. 1B. Kaplan-Meier survival analysis exhibited that patients in LINC00893 low expression group suffered a shorter survial time compared with those in LINC00893 high expression group (Fig. 1D). In addition, The statistical results of the relationship between LINC00893 and PCa clinicopathological indicators demonstrated that the LINC00893 expression was closely associated with TNM staging and distant metastasis, but has no relation with the patients' age, gender, tumor size and tumor differentiation degree (Table 1). Collectively, The above results indicated that LINC00893 functions as an anti-oncogenic factors in PCa.

\section{LINC00893 inhibits the proliferation, migration, invasion as well as EMT of PCa cells}

To further explore the function of LINC00893 in PCa cells, we conducted gain-of-function assays. PCa cells PC-3 and LNCaP with the lowest LINC00893 expression were transfected with pcDNA3.1-LINC00893 overexpression plasmids or matched controls. RT-qPCR was implemented to detect the overexpression efficiency, and the results identified that pcDNA3.1-LINC00893 plasmids could effectively overexpress LINC00893 in cells compared with matched controls (Fig. 2A). Subsequently, CCK-8 cell proliferation experiment and EDU experiment results demonstrated that LINC00893 inhibited the cell proliferation ability (Fig. 2B and C). We also performed colony formation assays, Transwell assays and the results revealed that LINC00893 weakened the ability of cell colony formation as well as cell metastasis (Fig. 2D, $E$ and $F$ ). In addition, we confirmed the effect of LINC00893 on EMT. LINC00893 significantly downregulated mesenchymal cell markers $\mathrm{N}$-cadherin and vimentin, whereas up-regulated epithelialcell marker E-cadherin (Fig. 2G). Together, the above results implied that LINC00893 inhibits the malignant phenotype of PCa cells as an anti-oncogenic fator.

\section{LINC00893 sponges miR-3173-5p and inhibits its expression}

To confirm the location of LINC00893 in cells, we performed nuclear and cytoplasmic separation experiment. We identified that LINC00893 was mainly located in cytoplasm (Fig. 3A). To unveil the underlying mechanism of LINC00893 regulaing the progresson of $\mathrm{PCa}$, we predicted the binding site between LINC00893 and miR-3173-5p through the Starbase database (Fig. 3B). To investigate the functional interaction between LINC00893 and miR-3173-5p, we conducted dual-luciferase reporter assays and the results demonstrated that miR-3173-5p inhibited the luciferase activity of pmirGLO-wide type (WT)-LINC00893 reporter with WT binding site of miR-3173-5p and LINC00893, but has no effect on pmirGLO-mutant (MUT)-LINC00893 reporter with MUT binding site (Fig. 3C). Furthermore, we also performed RNA Immunoprecipitation (RIP), anti-IgG antibody and anti-Ago2 antibody were used to pull down endogenous LINC00893 and miR-3173-5p (Ago2 is the indicator protein of IncRNA acting as a sponge and IgG as a negative control). Next, RT-qPCR results showed that anti-Ago2 antibody enriched more LINC00893 and miR-3173-5p compared with anti-lgG antibody (Fig. 3D). Consistently, RNA pulldown assay confirmed that Biotin-labeled miR-3173-5p probe enriched more LINC00893 than controls (Fig. 3E). We also assessed the miR-3173-5p expression in PCa tissues as well as matched paracancerous tissues employing RT-qPCR and found that the miR-3173-5p expression was up-regulated in PCa tissues (Fig. 3F). To further verified the correlation of LINC00893 and miR-3173-5p, we performed spearman correlation analysis and unveiled that LINC00893 negatively correlated with miR-3173-5p (Fig. 3G). In addition, We verified that miR-3173-5p expression was elevated in PCa cell lines PC-3, DU145, 
VCaP and LNCaP compared with that in human normal prostate epithelial cell line RWPE-1 (Fig. 3H). Lastly, we conducted gain-of-fuction experiments through overexpressing LINC00893 using pcDNA3.1LINC00893 overexpression plasmids and found that LINC00893 inhibited miR-3173-5p expression in PCa cells PC-3 and LNCaP (Fig. 3I). Taken together, the above results confirmed that LINC00893 functions as a sponge of miR-3173-5p and inhibit its expression.

\section{MiR-3173-5p mediates the anti-oncogenic role of LINC00893 in PCa}

To further determine the mediation role of miR-3173-5p in LINC00893 regulating PCa progression, we treated PC-3 and LNCaP cells under the following different conditions: pcDNA, pcDNA-LINC00893, pcDNA-LINC00893 + miR-3173-5p-mimic. Subsequently, we performed CCK-8 cell proliferation assay and EdU assay to assess the proliferation ability of cells, conducted colony formation assays to detect the colony formation ablity, Transwell assay and WB were used to detect migration, invation and EMT of cells. The results confirmed that overexpression of LINC00893 inhibited the abilities of cell proliferation and colony formation (Fig. 4A-C), reduced cell migration (Fig. 4D), cell invation (Fig. 4E) as well as EMT (Fig. 4F), but when miR-3173-5p was overexpressed, LINC00893-inhibited cell malignant phenotype was partly rescued. Collectively, The above results supported the notion that LINC00893 regulates PCa progression through sponging miR-3173-5p.

\section{miR-3173-5p targets 3'UTR region of SOCS3 mRNA}

To investigate the target of miR-3173-5p, we predicted and found the binding sites between miR-3173-5p and SOCS3 mRNA 3'UTR through starbase database. Subsequently, we confirmed the interaction of miR3173-5p and SOCS3 mRNA 3'UTR. The results proved that miR-3173-5p inhibit the luciferase activity of pmirGLO-WT-SOCS3 reporter, but has no effect on pmirGLO-MUT-SOCS3 reporter (Fig. 5A). Consistently, overexpression of miR-3173-5p inhibited the SOCS3 protein expression (Fig. 5B). Furthermore, we implement loss-of-function experiment through transfecting miR-3173-5p-inhibitor or miR-NC. Our subsequent results showed that miR-3173-5p inhibitor suppressed miR-3173-5p expression (Fig. 5C) and further increased the expression of SOCS3 protein (Fig. 5D). In addition, overexpression of LINC00893 increased SOCS3 protein level, while miR-3173-5p-mimic could partially reduced SOCS3 protein level upregulated by LINC00893 (Fig. 5E). We also assessed the SOCS3 protein level in PCa cell lines PC-3, DU145, VCaP and LNCaP as well as human normal prostate epithelial cell line RWPE-1 and found that SOCS3 protein level was reduced in PCa cell lines, and the lowest SOCS3 protein level was found in PC-3 and LNCaP cells (Fig. 5F). Consistently, the SOCS3 mRNA expression was down-regulated compared with matched para-cancerous tissues (Fig. 5G). We also detected SOCS3 expression using immunohistochemistry (IHC) staining and found that the positive staining rate of SOCS3 in PCa tissues was reduced compared with that in matched para-cancerous tissues (Fig. 5H). In addition, spearman correlation coefficient analysis confrimed that SOCS3 expression was positively correlated with LINC00893 expression and negatively correlated with miR-3173-5p expression (Fig. $5 \mathrm{I}$ and $\mathrm{J}$ ). Taken together, our data indicated that miR-3173-5p binds to 3'UTR of SOCS3 mRNA and inhibits its expression.

\section{Silencing SOCS3 partially alleviates the inhibitory effect of silencing miR-3173-5p on malignant phenotype of PCa}


To confirm the mediation role of SOCS3 in miR-3173-5p regulating malignant phenotype of PCa. PCa cells PC-3 and LNCaP were transfected with siRNA targeting SOCS3 (si-SOCS3) and matched controls (si$\mathrm{NC}$ ), Western blot method was used to assess the interference efficiency and the results showed that siSOCS3 effectively reduces the SOCS3 expression compared with si-NC (Fig. 6A). We also treated PC-3 and LNCaP cells under following conditions: NC inhibitor, miR-3173-5p inhibitor, miR-3173-5p inhibitor + si-SOCS3. CCK-8 proliferation assay, EdU assay, colony formation assays, transwell asssay and western blot assay showed that silencing miR-3173-5p inhibited the cell abilities of proliferation, colony formation ability, I migration, invation and EMT, while silencing SOCS3 rescued the abilities of proliferation (Fig. 6B and C), colony formation (Fig. 6D), migration (Fig. 6E) and invation (Fig. 6F), as well as EMT (Fig. 6G) regulated by inhibition of miR-3173-5p. Collectively, the above results suggested that SOCS3 mediates the regulation of miR-3173-5p on the malignant phenotype of PCa cells.

\section{LINC00893 regulates the SOCS3/JAK2/STAT3 pathway through sponging miR-3173-5p.}

To further explore the signaling pathway regulated by LINC00893 sponge miR-3173-5p We treated PC-3 and LNCaP cells under following conditions: pcDNA3.1, pcDNA3.1-LINC00893, pcDNA3.1-LINC00893 + miR-3173-5p. Subsequently, we detected the protein levels of SOCS3, p-JAK2, JAK2, p-STAT3 and STAT3. The results illustrated that LINC00893 promoted SOCS3 expression and inhibited p-JAK2 and p-STAT3 expression, but had no effect on JAK2 and STAT3 expression. While miR-3173-5p mimic weakened the SOCS3 expression and enhanced p-JAK2 and p-STAT3 expression, but also didn't affect JAK2 and STAT3 protein levels (Fig. 7A and B). Collectively, SOCS3/JAK2/STAT3 pathway is the target of miR-3173-5p sponged by LINC00893.

\section{Overexpression of LINC00893 suppressed the proliferation of PCa cells in vivo.}

To further confirm the role of LINC00893 in regulating the progression of PCa in vivo, we conducted xenograft tumorigenesis assay in mice. The in vivo xenograft model was established through subcutaneous injection of PC-3- LINC00893 cells or PC-3- vector cells in mice. The assay showed that LINC00893 hindered the growth of PC-3 cells in mice (Fig. 8A and B). Subsequently, we extracted RNA from PC-3- LINC00893 tumor and PC-3-vector tumor and used RT-qPCR for RNA quantification, and found that compared with PC-3-vector tumor cells, the expression of LINC00893 and miR-3173-5p were upregulated and down-regulated in PC-3-LINC00893 tumor cells, respectively (Fig. 8C). Furthermore, the protein levels of SOCS3 and E-cadherin were up-regulated, while N-cadherin and vimentin were downregulated in PC-3-LINC00893 tumor cells than that in PC-3-vector tumor cells (Fig. 8D). Consistently, We also found that LINC00893 negatively regulated the $\mathrm{KI}-67$ protein expression but positively regulated SOCS3 protein expression in xenograft tumor tissues through IHC staining (Fig. 8E). Taken together, The above results supported the notion that LINC00893 inhibites a wide range of malignant phenotype including proliferation, EMT and metastasis in vivo.

\section{Discussion}


In summary, we found that LINC00893 was lowly expressed in PCa tissues and cell lines, and the LINC00893 expression was closely associated with the TNM staging and distant metastasis of PCa. Patients with low LINC00893 expression bore a poor prognosis. These data suggests the anti-oncogenic effect of LINC00893. To further verify the regulatory role of LINC00893 in the progression of PCa, we conducted a gain-of-function experiment through overexpressing LINC00893 and found that LINC00893 facilitated the growth, EMT as well as metastasis of PCa cells in vitro and in vivo. Subsequently, to explore the target by which LINC00893 regulates the malignant phenotype of PCa cells, we predicted and found the binding site of LINC00893 on miR-3173-5p through the Starbase database, which was verified by a spectrum of assays including Dual-Luciferase reporter, RIP as well as RNA pull-down. Based on these data, we also found LINC00893 was negatively correlated with miR-3173-5p in PCa tissues. Further rescue assay confirmed that miR-3173-5p mediated the effect of LINC00893 on inhibiting PCa progression. The above data indicates the fact that LINC00893 functions as a miR-3173-5p sponge and inhibits its expression. To further search for the downstream target of miR-3173-5p, we analyzed the Starbase database once again and identified a binding site of miR-3173-5p in the 3'UTR region of SOCS3 mRNA, which was confirmed by a Dual-Luciferase reporter assay. Furthermore, SOCS3 expression was negatively regulated by miR-3173-5p and SOCS3 mediated the regulation of miR-3173-5p on the malignant phenotype of PCa cells. In conclusion, our study reveals the novel role of LINC00893 in the progression of PCa and a novel mechanism by which LINC00893 sponges miR-3173-5p and further regulate the SOCS3/JAK2/STAT3 axis.

Recent studies have found that the IncRNA AATBC enhances the proliferation and metastasis of PCa cells through its sponge effect on miR-1245b-5p,thereby facilitating the expression of CASK [22]. The IncRNA CRNDE targets miRNA-146a-5p and further regulates the progression of PCa [46]. The IncRNA AFAP1-AS1 promotes the PCa progression through the miR-15b/IGF1R axis [47]. The above studies have shown that the LncRNA/miRNA axis exerts a prominent role in the PCa progression. LINC00893 is a newly discovered IncRNA. At present, there are few studies on it in cancer. Recent studies have shown that LINC00893 is down-regulated in thyroid cancer (THCA) tissues and inhibits the growth and metastasis of THCA cells through stablizing PTEN and further inhibiting the AKT pathway [48]. However, the function and regulation mechanism of LINC00893 in PCa remains to be investigated. At present, the research on miR-3173 in cancer is rarely involved. The only studies showed that miR-3173 is implicated in the progression of ovarian carcinoma [49] and B-cell acute lymphoblastic leukemia[50]. However, whether mir-3173 is involved in the development of PCA remains to be further studied. Consistent with the function of LINC00893 in THCA, LINC00893 is down-regulated in PCa tissues as well as cell lines, which leads to lower survival rates. These data proved a anti-oncpgenic role of LINC00893. Interestingly, we found the downstream target miR-3173 of LINC00893 through the Starbase database. LINC00893 functions as a miR-3173 sponge and adsorbs it, thereby regulating the proliferation, EMT and metastasis of PCa cells. In conclusion, our study clarified the novel functional role of LINC00893 and its downstream target.

The JAK2/STAT3 signaling pathway is associated with the progression of various tumors. In PCa, JAK2/STAT3 pathway regulates the malignant phenotype of cancer cells through regulation of metabolic 
pathways [51]. Furthermore, JAK2/STAT3 signaling pathway is also the crucial mediator for the antitumor effects of Scoparone [52], $\beta$-citric acid [53] and Atractylenolide II [54]. In other cancers, the JAK2/STAT3 signaling pathway is also implicated in cancer progression. In lung adenocarcinoma, JAK2/STAT3 pathway is also implicated in EMT and metastasis of cancer cells [55]. In melanoma, the anti-tumor effect of brevilin A also depends on the JAK2/STAT3 pathway [56]. The above studies suggests a crucial oncogenic role of JAK2/STAT3 signaling pathway. Therefore, JAK2 inhibitor combined with methylsulfonylmethane synergistically effectively prevent the progression of Bladder Cancer [57]. As a negative regulator of JAK2/STAT3 signaling pathway, SOCS3 also occupies an irreplaceable position in PCa. For example, miR-2909 regulates the ISGylation system by inhibiting SOCS3 [58]. SOCS3 affects the sensitivity of PCa cells to the targeted anticancer drug Enzalutamide [59]. SOCS3 suppresses the growth and metastasis of PCa cells and prolongs the survival time of PCa patients [60]. Take together, the abnormal expression of SOCS3 is implicated in the progression of PCa and the prognosis of patients [61]. In addition, it has been observed that SOCS3 is inhibited in PCa, which leads to overactivation of the JAK/STAT3 signaling pathway [62]. Our research supports the function of the SOCS3/JAK2/STAT3 signaling axis in the PCa progression. More importantly, we found the upstream regulator miR-3173-5p and LINC00893 of SOCS3/JAK2/STAT3 axis. LINC00893 and miR-3173-5p functions as the upstream regulators of SOCS3/JAK2/STAT3 axis to jointly regulate the malignant progression of PCa. LINC00893 inhibits the expression of miR-3173-5p depends on its sponge effect, which eliminates the suppressive role of miR-3173-5p in SOCS3 expression and inhibites the JAK2/STAT3 signaling pathway and ultimately prevents the proliferation, EMT and metastasis of PCa cells. The combination of specific inhibitors of the JAK2/STAT3 signaling pathway with LINC00893 and miR-3173-5p may lay a theoretical foundation for the targeted therapy of PCa.

\section{Conclusions}

In conclusion, this study clarified the role and mechanism of LINC00893 in regulating the PCa progression. LINC00893 functions as an anti-oncogenic molecule to suppress the growth, EMT and metastasis of PCa cells, thereby hindering the progression of PCa. Consistently, corresponding clinical evidence also indicated that LINC00893 is a anti-oncogrnic molecule in PCa patients. In terms of mechanism, LINC00893 binds to miR-3173-5p as ceRNA, thereby impairing the inhibitory role of miR3173-5p in SOCS3, and further inhibiting the JAK2/STAT3 signaling pathway. Our research has enriched the molecular network of LINC00893 regulating the progression of $\mathrm{PCa}$, and provided a theoretical foundation for PCa targeted therapy.

\section{Declarations}

Acknowledgements

Not applicable

\section{Authors' contributions}


C.G..Y. carried out most of the experiments and analyzed the data. Y. F. wrote the manuscript and prepared the figures. Y.Z. and L.P.L. conducted some experiments. G. G. designed the project and revised the manuscript.

\section{Funding}

Not applicable

\section{Availability of data and materials}

All data and materials were contained in the final version manuscript.

\section{Ethics approval and consent to participate}

This study has been approved by the Medical Ethics Committee of The Third Medical Center of Chinese People's Liberation Army General Hospital from the collection of samples to the conduction of all research procedures.

\section{Consent for publication}

Not applicable.

\section{Competing interests}

The authors declare no conflict of interests.

\section{Author details}

Department of Urology, The Third Medical Center, Chinese People's Liberation Army General Hospital. No 69 , Yongding Road, Haidian District, Beijing 100039, China.

\section{References}

1. Schatten H: Brief Overview of Prostate Cancer Statistics, Grading, Diagnosis and Treatment Strategies. Advances in experimental medicine and biology 2018, 1095:1-14.

2. Gomella LG: Prostate Cancer Statistics: Anything You Want Them To Be. The Canadian journal of urology 2017, 24(1):8603-8604.

3. Bostwick DG, Burke HB, Djakiew D, Euling S, Ho SM, Landolph J, Morrison H, Sonawane B, Shifflett T, Waters DJ et al: Human prostate cancer risk factors. Cancer 2004, 101(10 Suppl):2371-2490.

4. Pienta KJ, Esper PS: Risk factors for prostate cancer. Annals of internal medicine 1993, 118(10):793803. 
5. Kolonel LN, Altshuler D, Henderson BE: The multiethnic cohort study: exploring genes, lifestyle and cancer risk. Nature reviews Cancer 2004, 4(7):519-527.

6. Dagnelie PC, Schuurman AG, Goldbohm RA, Van den Brandt PA: Diet, anthropometric measures and prostate cancer risk: a review of prospective cohort and intervention studies. BJU internationa/2004, 93(8):1139-1150.

7. Evans AJ: Treatment effects in prostate cancer. Modern pathology : an official journal of the United States and Canadian Academy of Pathology, Inc 2018, 31(S1):S110-121.

8. Lee JT: Epigenetic regulation by long noncoding RNAs. Science 2012, 338(6113):1435-1439.

9. Yang S, Sun Z, Zhou Q, Wang W, Wang G, Song J, Li Z, Zhang Z, Chang Y, Xia K et al: MicroRNAs, long noncoding RNAs, and circular RNAs: potential tumor biomarkers and targets for colorectal cancer. Cancer management and research 2018, 10:2249-2257.

10. Gao C, Lu W, Lou W, Wang L, Xu Q: Long noncoding RNA HOXC13-AS positively affects cell proliferation and invasion in nasopharyngeal carcinoma via modulating miR-383-3p/HMGA2 axis. Journal of cellular physiology 2019, 234(8):12809-12820.

11. Huarte M: The emerging role of IncRNAs in cancer. Nature medicine 2015, 21(11):1253-1261.

12. Bhat SA, Ahmad SM, Mumtaz PT, Malik AA, Dar MA, Urwat U, Shah RA, Ganai NA: Long non-coding RNAs: Mechanism of action and functional utility. Non-coding RNA research 2016, 1(1):43-50.

13. Reddy KB: MicroRNA (miRNA) in cancer. Cancer cell international 2015, 15:38.

14. Luo ZF, Peng Y, Liu FH, Ma JS, Hu G, Lai SL, Lin H, Chen JJ, Zou GM, Yan Q et al: Long noncoding RNA SNHG14 promotes malignancy of prostate cancer by regulating with miR-5590-3p/YY1 axis. European review for medical and pharmacological sciences 2020, 24(9):4697-4709.

15. Dong ZQ, Guo ZY, Xie J: The IncRNA EGFR-AS1 is linked to migration, invasion and apoptosis in glioma cells by targeting miR-133b/RACK1. Biomedicine \& pharmacotherapy = Biomedecine \& pharmacotherapie 2019, 118:109292.

16. Guo F, Zhu X, Zhao Q, Huang Q: miR5893p sponged by the IncRNA TINCR inhibits the proliferation, migration and invasion and promotes the apoptosis of breast cancer cells by suppressing the Akt pathway via IGF1R. International journal of molecular medicine 2020, 46(3):989-1002.

17. Ma JY, Liu SH, Chen J, Liu Q: Metabolism-related long non-coding RNAs (IncRNAs) as potential biomarkers for predicting risk of recurrence in breast cancer patients. Bioengineered 2021, 12(1):37263736 . 
18. Krasniqi E, Sacconi A, Marinelli D, Pizzuti L, Mazzotta M, Sergi D, Capomolla E, Donzelli S, Carosi M, Bagnato A et al: MicroRNA-based signatures impacting clinical course and biology of ovarian cancer: a miRNOmics study. Biomarker research 2021, 9(1):57.

19. Ballantyne MD, McDonald RA, Baker AH: IncRNA/MicroRNA interactions in the vasculature. Clinical pharmacology and therapeutics 2016, 99(5):494-501.

20. Jiang X, Guo S, Zhang Y, Zhao Y, Li X, Jia Y, Xu Y, Ma B: LncRNA NEAT1 promotes docetaxel resistance in prostate cancer by regulating ACSL4 via sponging miR-34a-5p and miR-204-5p. Cellular signalling 2020, 65:109422.

21. Zhang C, Wang GX, Fu B, Zhou XC, Li Y, Li YY: LncRNA CASC15 promotes migration and invasion in prostate cancer via targeting miR-200a-3p. European review for medical and pharmacological sciences 2019, 23(19):8303-8309.

22. Zhang W, Liu Q, Zhao J, Wang T, Wang J: Long Noncoding RNA AATBC Promotes the Proliferation and Migration of Prostate Cancer Cell Through miR-1245b-5p/CASK Axis. Cancer management and research 2021, 13:5091-5100.

23. Balko JM, Schwarz LJ, Luo N, Estrada MV, Giltnane JM, Davila-Gonzalez D, Wang K, Sanchez V, Dean PT, Combs SE et al: Triple-negative breast cancers with amplification of JAK2 at the 9p24 locus demonstrate JAK2-specific dependence. Science translational medicine 2016, 8(334):334ra353.

24. Duru N, Fan M, Candas D, Menaa C, Liu HC, Nantajit D, Wen Y, Xiao K, Eldridge A, Chromy BA et al: HER2-associated radioresistance of breast cancer stem cells isolated from HER2-negative breast cancer cells. Clinical cancer research : an official journal of the American Association for Cancer Research 2012, 18(24):6634-6647.

25. Teng TS, Lin B, Manser E, Ng DC, Cao X: Stat3 promotes directional cell migration by regulating Rac1 activity via its activator betaPIX. Journal of cell science 2009, 122(Pt 22):4150-4159.

26. Xiong H, Zhang ZG, Tian XQ, Sun DF, Liang QC, Zhang YJ, Lu R, Chen YX, Fang JY: Inhibition of JAK1, 2/STAT3 signaling induces apoptosis, cell cycle arrest, and reduces tumor cell invasion in colorectal cancer cells. Neoplasia 2008, 10(3):287-297.

27. Lo HW, Cao X, Zhu H, Ali-Osman F: Constitutively activated STAT3 frequently coexpresses with epidermal growth factor receptor in high-grade gliomas and targeting STAT3 sensitizes them to Iressa and alkylators. Clinical cancer research : an official journal of the American Association for Cancer Research 2008, 14(19):6042-6054.

28. Lv Y, Song G, Li P: Correlation of SOCS-1 gene with onset and prognosis of breast cancer. Oncology letters 2018, 16(1):383-387. 
29. Ram PA, Waxman DJ: SOCS/CIS protein inhibition of growth hormone-stimulated STAT5 signaling by multiple mechanisms. The Journal of biological chemistry 1999, 274(50):35553-35561.

30. Akhtar LN, Benveniste EN: Viral exploitation of host SOCS protein functions. Journal of virology 2011, 85(5):1912-1921.

31. Kotaja N, Karvonen U, Janne OA, Palvimo JJ: PIAS proteins modulate transcription factors by functioning as SUMO-1 ligases. Molecular and cellular biology 2002, 22(14):5222-5234.

32. Sasaki A, Yasukawa H, Suzuki A, Kamizono S, Syoda T, Kinjyo I, Sasaki M, Johnston JA, Yoshimura A: Cytokine-inducible SH2 protein-3 (CIS3/SOCS3) inhibits Janus tyrosine kinase by binding through the Nterminal kinase inhibitory region as well as $\mathrm{SH} 2$ domain. Genes to cells : devoted to molecular \& cellular mechanisms 1999, 4(6):339-351.

33. Vainchenker W, Constantinescu SN: JAK/STAT signaling in hematological malignancies. Oncogene 2013, 32(21):2601-2613.

34. Mahony R, Ahmed S, Diskin C, Stevenson NJ: SOCS3 revisited: a broad regulator of disease, now ready for therapeutic use? Cellular and molecular life sciences : CMLS 2016, 73(17):3323-3336.

35. Hu Y, Luo X, Zhou J, Chen S, Gong M, Deng Y, Zhang H: Piperlongumine inhibits the progression of osteosarcoma by downregulating the SOCS3/JAK2/STAT3 pathway via miR-30d-5p. Life sciences 2021, 277:119501.

36. Attia YM, Tawfiq RA, Gibriel AA, Ali AA, Kassem DH, Hammam OA, Elmazar MM: Activation of FXR modulates SOCS3/Jak2/STAT3 signaling axis in a NASH-dependent hepatocellular carcinoma animal model. Biochemical pharmacology 2021, 186:114497.

37. Wang H, Zhan M, Liu Q, Wang J: Glycochenodeoxycholate promotes the metastasis of gallbladder cancer cells by inducing epithelial to mesenchymal transition via activation of SOCS3/JAK2/STAT3 signaling pathway. Journal of cellular physiology 2020, 235(2):1615-1623.

38. Kong PZ, Yang F, Li L, Li XQ, Feng YM: Decreased FOXF2 mRNA expression indicates early-onset metastasis and poor prognosis for breast cancer patients with histological grade II tumor. PloS one 2013, 8(4):e61591.

39. Sun L, Zheng W, Liu QD, Ge L: Valproic Acid Protects Chondrocytes from LPS-Stimulated Damage via Regulating miR-302d-3p/ITGB4 Axis and Mediating the PI3K-AKT Signaling Pathway. Frontiers in molecular biosciences 2021, 8:633315.

40. Zhong Z, Liang S, Sanchez-Lopez E, He F, Shalapour S, Lin XJ, Wong J, Ding S, Seki E, Schnabl B et al: New mitochondrial DNA synthesis enables NLRP3 inflammasome activation. Nature 2018, 560(7717):198-203. 
41. Yu F, Lin Y, Xu X, Liu W, Tang D, Zhou X, Wang G, Zheng Y, Xie A: Knockdown of GSG2 inhibits prostate cancer progression in vitro and in vivo. International journal of oncology 2020, 57(1):139-150.

42. Yu Y, Xiao CH, Tan LD, Wang QS, Li XQ, Feng YM: Cancer-associated fibroblasts induce epithelialmesenchymal transition of breast cancer cells through paracrine TGF-beta signalling. British journal of cancer 2014, 110(3):724-732.

43. Chen J, Huang X, Wang W, Xie H, Li J, Hu Z, Zheng Z, Li H, Teng L: LncRNA CDKN2BAS predicts poor prognosis in patients with hepatocellular carcinoma and promotes metastasis via the miR-1535p/ARHGAP18 signaling axis. Aging 2018, 10(11):3371-3381.

44. Liu S, Duan K, Zhang X, Cao X, Wang X, Meng F, Liu H, Xu B, Wang X: Circ_0081001 down-regulates miR-494-3p to enhance BACH1 expression and promotes osteosarcoma progression. Aging 2021, 13.

45. Zhang C, Cao J, Lv W, Mou H: CircRNA_100395 Carried by Exosomes From Adipose-Derived Mesenchymal Stem Cells Inhibits the Malignant Transformation of Non-Small Cell Lung Carcinoma Through the miR-141-3p-LATS2 Axis. Frontiers in cell and developmental biology 2021, 9:663147.

46. Fu D, Zang L, Li Z, Fan C, Jiang H, Men T: Long non-coding RNA CRNDE regulates the growth and migration of prostate cancer cells by targeting microRNA-146a-5p. Bioengineered 2021, 12(1):2469-2479.

47. Liu B, Jiang HY, Yuan T, Zhou WD, Xiang ZD, Jiang QQ, Wu DL: Long non-coding RNA AFAP1-AS1 facilitates prostate cancer progression by regulating miR-15b/IGF1R axis. Current pharmaceutical design 2021.

48. Li S, Zhang Y, Dong J, Li R, Yu B, Zhao W, Liu J: LINC00893 inhibits papillary thyroid cancer by suppressing AKT pathway via stabilizing PTEN. Cancer biomarkers : section A of Disease markers 2021, 30(3):277-286.

49. Barbier J, Chen X, Sanchez G, Cai M, Helsmoortel M, Higuchi T, Giraud P, Contreras X, Yuan G, Feng Z et al: An NF90/NF110-mediated feedback amplification loop regulates dicer expression and controls ovarian carcinoma progression. Cell research 2018, 28(5):556-571.

50. Tian L, Cao J, Ji Q, Zhang C, Qian T, Song X, Huang B, Tian X: The downregulation of miR-3173 in Bcell acute lymphoblastic leukaemia promotes cell invasion via PTK2. Biochemical and biophysical research communications 2017, 494(3-4):569-574.

51. Marrocco I, Altieri F, Rubini E, Paglia G, Chichiarelli S, Giamogante F, Macone A, Perugia G, Magliocca FM, Gurtner A et al: Shmt2: A Stat3 Signaling New Player in Prostate Cancer Energy Metabolism. Cells $2019,8(9)$.

52. Kim JK, Kim JY, Kim HJ, Park KG, Harris RA, Cho WJ, Lee JT, Lee IK: Scoparone exerts anti-tumor activity against DU145 prostate cancer cells via inhibition of STAT3 activity. PloS one 2013, 8(11):e80391. 
53. Bao X, Zhu J, Ren C, Zhao A, Zhang M, Zhu Z, Lu X, Zhang Y, Li X, Sima X et al: beta-elemonic acid inhibits growth and triggers apoptosis in human castration-resistant prostate cancer cells through the suppression of JAK2/STAT3/MCL-1 and NF-kB signal pathways. Chemico-biological interactions 2021, 342:109477.

54. Wang J, Nasser MI, Adlat S, Ming Jiang M, Jiang N, Gao L: Atractylenolide II Induces Apoptosis of Prostate Cancer Cells through Regulation of AR and JAK2/STAT3 Signaling Pathways. Molecules 2018, 23(12).

55. Huo SF, Shang WL, Yu M, Ren XP, Wen HX, Chai CY, Sun L, Hui K, Liu LH, Wei SH et al: STEAP1 facilitates metastasis and epithelial-mesenchymal transition of lung adenocarcinoma via the JAK2/STAT3 signaling pathway. Bioscience reports 2020, 40(6).

56. Su T, Wang YP, Wang XN, Li CY, Zhu PL, Huang YM, Yang ZY, Chen SB, Yu ZL: The JAK2/STAT3 pathway is involved in the anti-melanoma effects of brevilin A. Life sciences 2020, 241:117169.

57. Joung YH, Na YM, Yoo YB, Darvin P, Sp N, Kang DY, Kim SY, Kim HS, Choi YH, Lee HK et al: Combination of AG490, a Jak2 inhibitor, and methylsulfonylmethane synergistically suppresses bladder tumor growth via the Jak2/STAT3 pathway. International journal of oncology 2014, 44(3):883-895.

58. Ayub SG, Kaul D: miR-2909 regulates ISGylation system via STAT1 signalling through negative regulation of SOCS3 in prostate cancer. Andrology 2017, 5(4):790-797.

59. Handle F, Erb HH, Luef B, Hoefer J, Dietrich D, Parson W, Kristiansen G, Santer FR, Culig Z: SOcS3 Modulates the Response to Enzalutamide and Is Regulated by Androgen Receptor Signaling and CpG Methylation in Prostate Cancer Cells. Molecular cancer research : MCR 2016, 14(6):574-585.

60. Kneitz B, Krebs M, Kalogirou C, Schubert M, Joniau S, van Poppel H, Lerut E, Kneitz S, Scholz CJ, Strobel $\mathrm{P}$ et al: Survival in patients with high-risk prostate cancer is predicted by miR-221, which regulates proliferation, apoptosis, and invasion of prostate cancer cells by inhibiting IRF2 and SOCS3. Cancer research 2014, 74(9):2591-2603.

61. Zhu JG, Yuan DB, Chen WH, Han ZD, Liang YX, Chen G, Fu X, Liang YK, Chen GX, Sun ZL et al: Prognostic value of ZFP36 and SOCS3 expressions in human prostate cancer. Clinical \& translational oncology : official publication of the Federation of Spanish Oncology Societies and of the National Cancer Institute of Mexico 2016, 18(8):782-791.

62. Yoneda T, Kunimura N, Kitagawa K, Fukui Y, Saito H, Narikiyo K, Ishiko M, Otsuki N, Nibu KI, Fujisawa $\mathrm{M}$ et al: Overexpression of SOCS3 mediated by adenovirus vector in mouse and human castrationresistant prostate cancer cells increases the sensitivity to NK cells in vitro and in vivo. Cancer gene therapy 2019, 26(11-12):388-399.

\section{Table}




\section{Table 1}

The correlation between the expression of LINC00893 and the clinicopathological features of $\mathrm{PCa}$

\begin{tabular}{|c|c|c|c|}
\hline \multirow{2}{*}{ Variable } & \multicolumn{2}{|c|}{ LINC00893 } & \multirow{2}{*}{$P$ value } \\
\hline & $\operatorname{Low}(n=33)$ & $\operatorname{High}(n=33)$ & \\
\hline Age(years) & & & 0.614 \\
\hline$\leq 60$ & 19 & 21 & \\
\hline$>60$ & 14 & 12 & \\
\hline Sex & & & 0.492 \\
\hline Female & 6 & 4 & \\
\hline Male & 27 & 29 & \\
\hline Tumor size & & & 0.083 \\
\hline$\leq 5$ & 22 & 15 & \\
\hline$>5$ & 11 & 18 & \\
\hline TNM stage & & & 0.026 \\
\hline I/ II & 19 & 10 & \\
\hline III/IV & 14 & 23 & \\
\hline Distant metastasis & & & 0.048 \\
\hline Yes & 11 & 19 & \\
\hline No & 22 & 14 & \\
\hline Tumor differentiation & & & 0.138 \\
\hline High/Middle & 12 & 18 & \\
\hline Low & 21 & 15 & \\
\hline
\end{tabular}

\section{Figures}

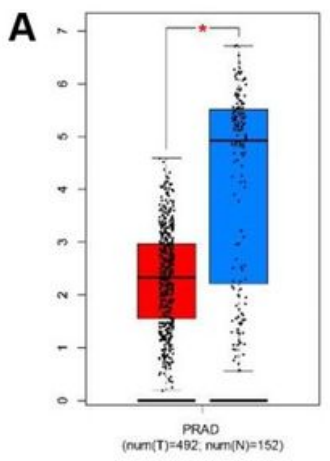

B

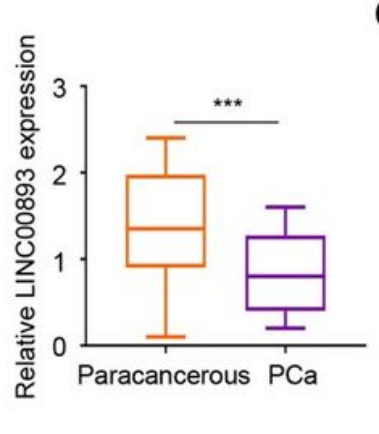

C

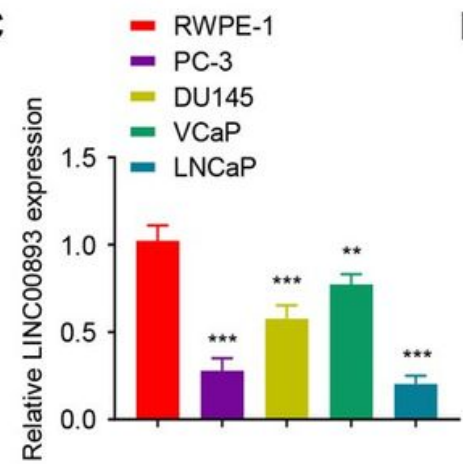

D

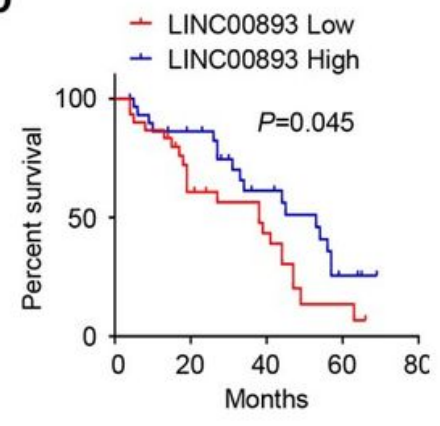

Figure 1 
LINC00893 expression in PCa tissues and cell lines is lower than that in their matched controls. A. LINC00893 expression in PCa tissues and matched para-cancerous tissues was mined in GSE73397_ GSE26910 dataset from TCGA database. B and C. LINC00893 expression levels in 66 pairs of PCa tissues and cell lines and their matched controls were detected by RT-qPCR. D. 66 patiens with PCa in Fig. 1B were assigned as LINC00893low $(n=33)$ and LINC00893high $(n=33)$ group according to the median value of LINC00893 expression, Kaplan-Meier survival analysis was used to evaluate the overall survival rate of patients in the two groups. Three independent assays were performed with three technical replicates. The error bars are defined as s.d. ${ }^{*}, P<0.05, * \star, P<0.01$, and $* \star \star, P<0.001$. 
A

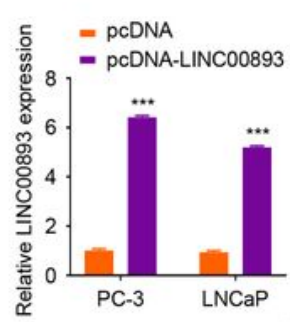

B

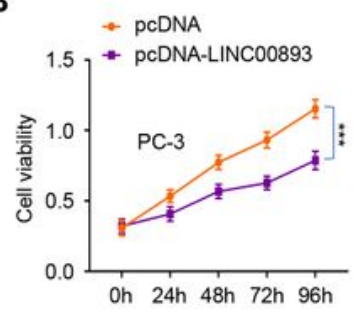

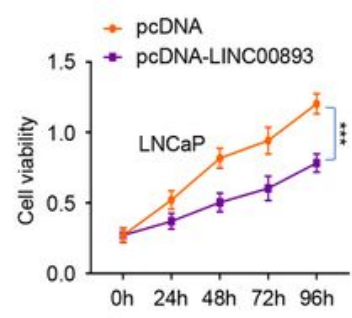

C
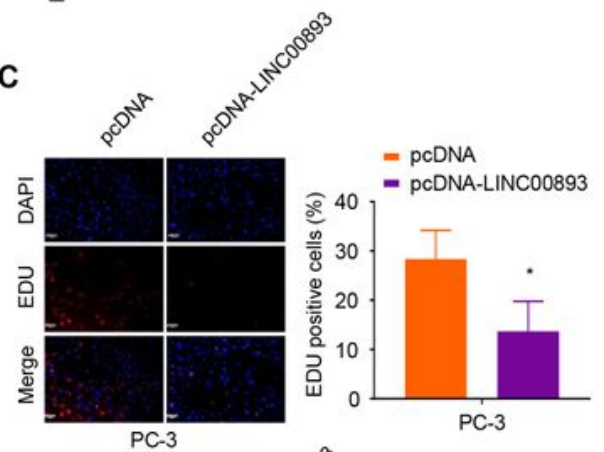

D
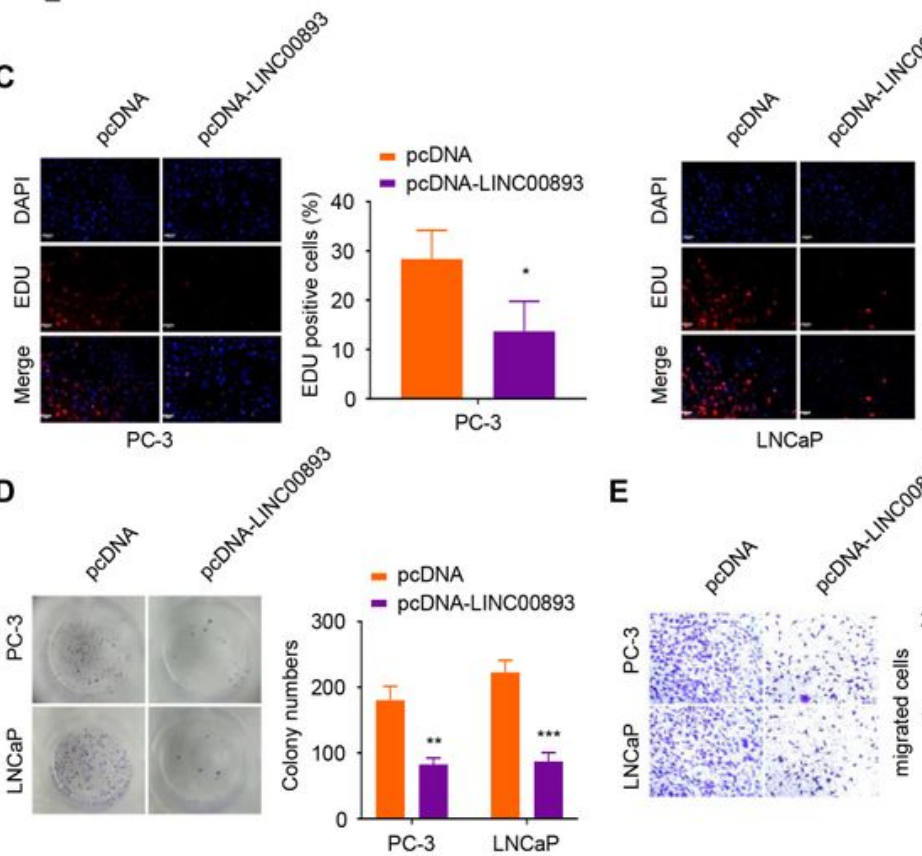

E

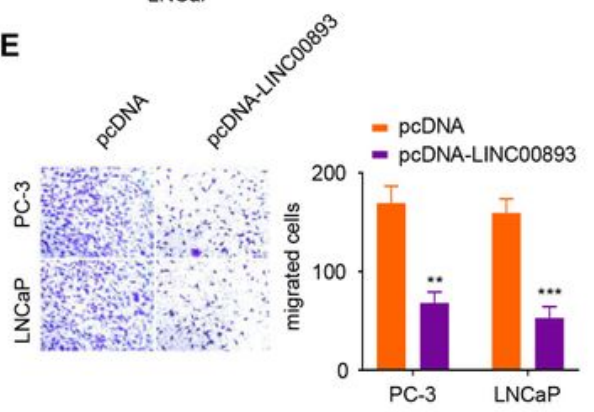

F

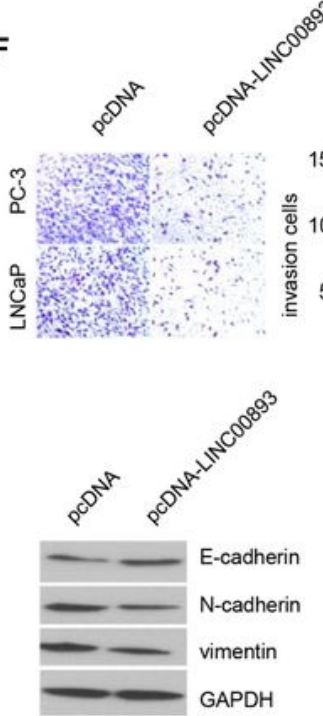

LNCaP
G

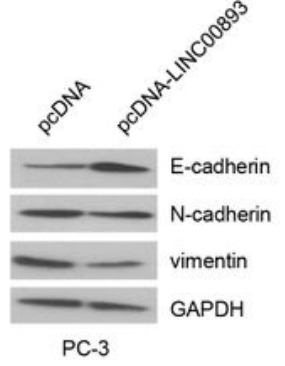

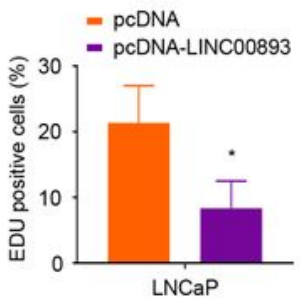

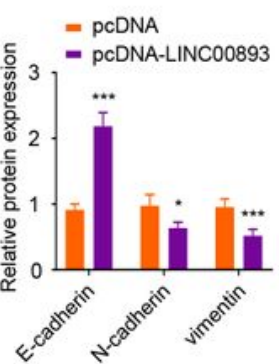

\section{Figure 2}

LINC00893 functions as an anti-oncogenic factor to inhibit the proliferation, migration, invasion and EMT of PCa cells. PCa cells PC-3 and LNCaP were transfected with pcDNA3.1-LINC00893 overexpression plasmids or pcDNA3.1-vector. A. the overexpression efficiency was tested by RT- qPCR. B and C. The cell

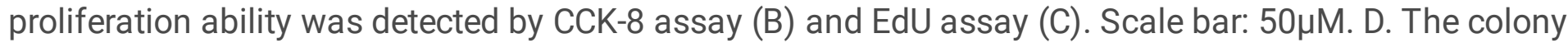
formation ability of cells was assessed by colony formation assay. E and F. The metastatic ability of cells 
was evaluated by cell migration (E) and invasion (F) assays. G. The protein expression levels of $\mathrm{E}-$ cadherin and mesenchymal cell markers $\mathrm{N}$-cadherin and vimentin were assessed by western bolt. Three independent assays were performed with three technical replicates. The error bars are defined as s.d. *, $P$ $<0.05$, **, $\mathrm{P}<0.01$, and $* * *, \mathrm{P}<0.001$.

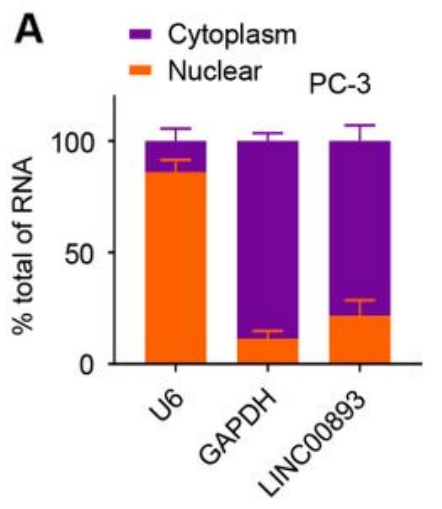

C

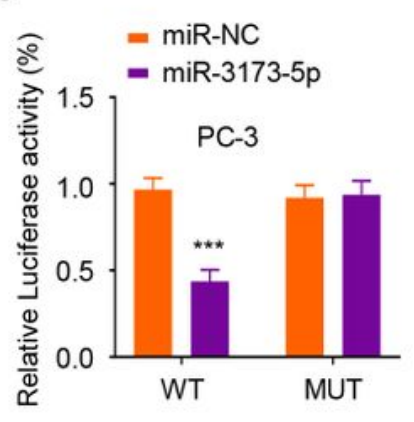

E

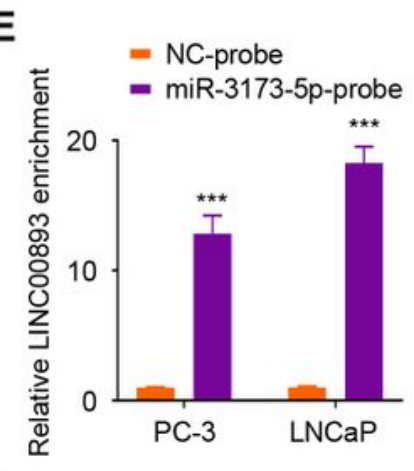

I

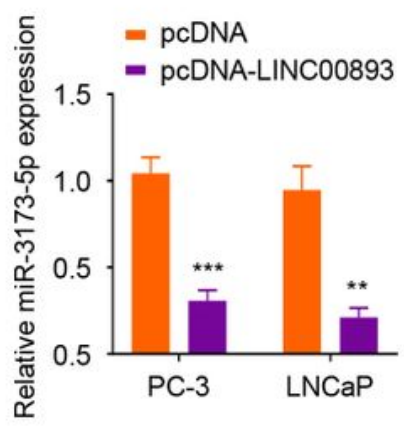

\section{B}

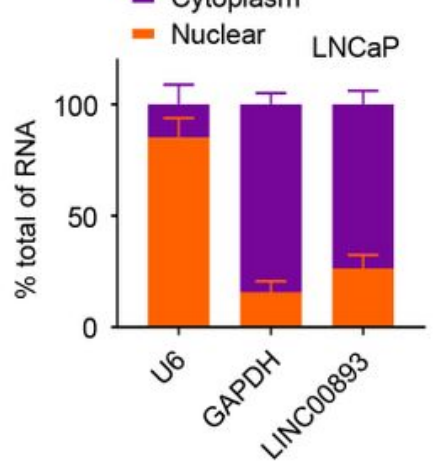

WT-LINC00893 5'-CUAGGCUGAGUCUUGCAGGGCU-3'

III II 1111111

hsa-miR-3173-5p 3'-UUUCCUCUUUUGUCCGUCCCGU-5'

MUT-LINC00893 5'-CUCACCUACAUCUUACGGCACAU-3'

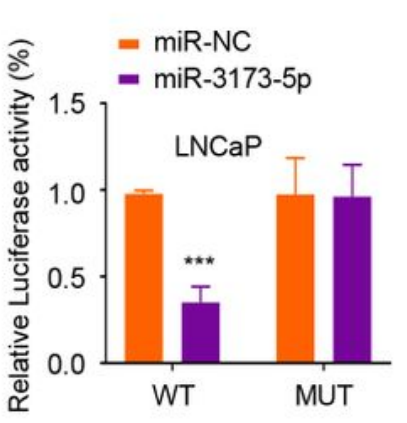

D

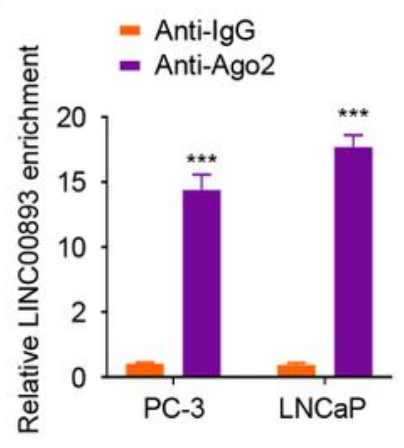

$\mathbf{F}$

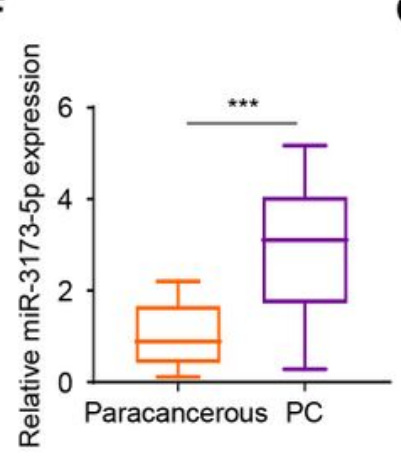

G

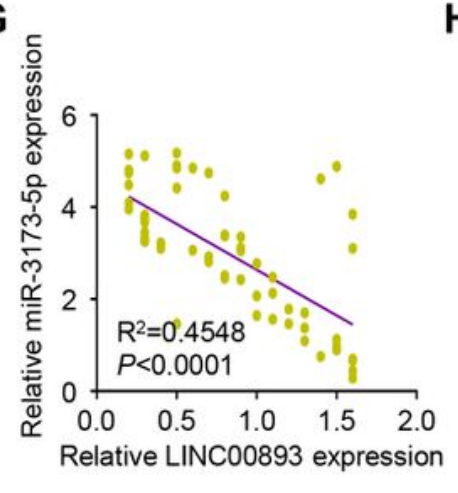

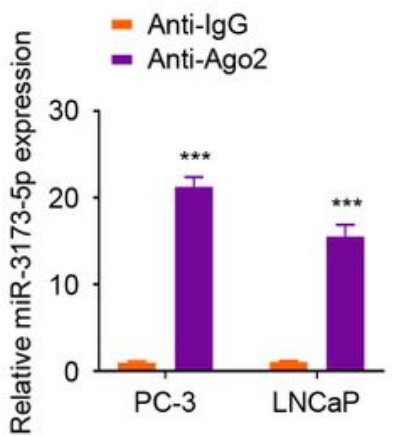

H

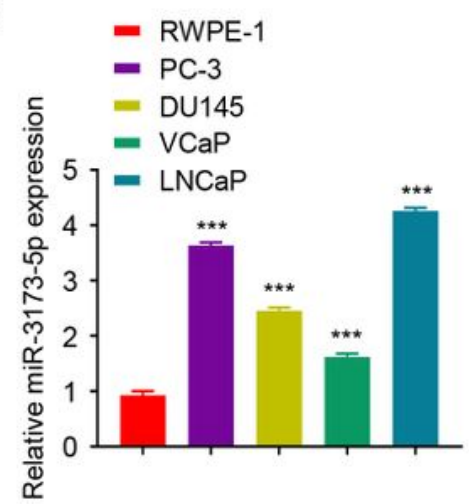

Figure 3 
LINC00893 binds to miR-3173-5p and inhibits its expression. A. The RNA located in the cytoplasm and nucleus of cells was separated using the RNA Subcellular Isolation kit. Subsequently, the LINC00893 expression levels in the cytoplasm and nucleus were detected by RT-qPCR. U6 small nuclear RNA and GAPDH were used as internal references for the nucleus and cytoplasm, respectively. $B$. The binding sites between LINC00893 and miR-3173-5p were searched from Starbase database. C. Dual-luciferase reporter recombination plasmids pmirGLO-WT-LINC00893/pmirGLO-MUT-LINC00893 and miR-3173-5p/miR-NC and renilla luciferase control plasmids were co-transfected into cells. The binding of LINC00893 to miR3173-5p was detected by the dual-luciferase reporter assay. Relative luciferase activity was normalized to renilla luciferase activity. $D$ and $E$. The interaction between LINC00893 and miR-3173-5p was assessed by RIP assay (D) and RNA pull-down assay (E). F. miR-3173-5p expression levels in 66 pairs of PCa tissues and matched para-cancerous tissues were assessed by RT-qPCR. G. The correlation between LINC00893 and miR-3173-5p was evaluated by Spearman correlation coefficient analysis. H. miR-3173-5p expression levels in PCa cell lines PC-3, DU145, VCaP and LNCaP and human normal prostate epithelial cell line RWPE-1 were detected by RT-qPCR. I. miR-3173-5p expression levels after overexpression of LINC00893 was assessed by RT-qPCR. Three independent assays were performed with three technical replicates. The error bars are defined as s.d. *, $P<0.05, * *, P<0.01$, and $* \star *, P<0.001$. 


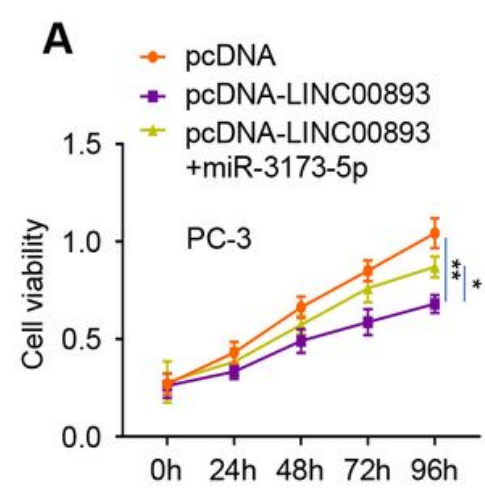

C

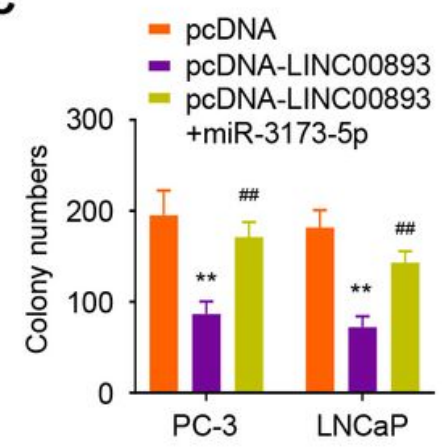

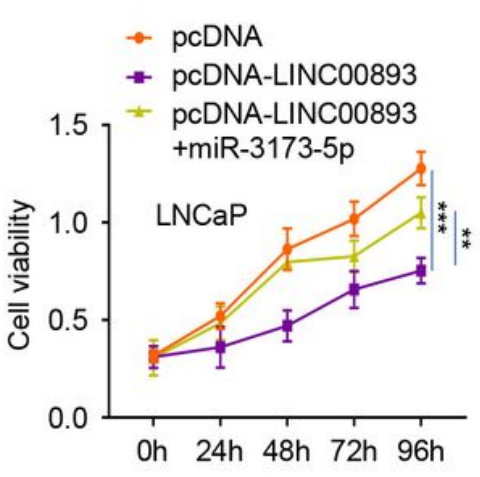

D

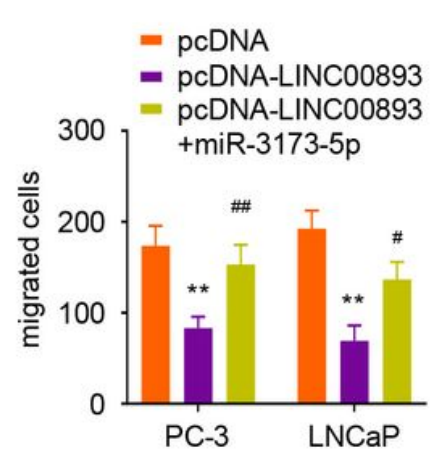

B

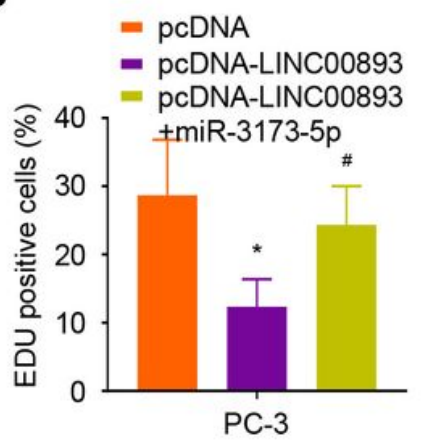

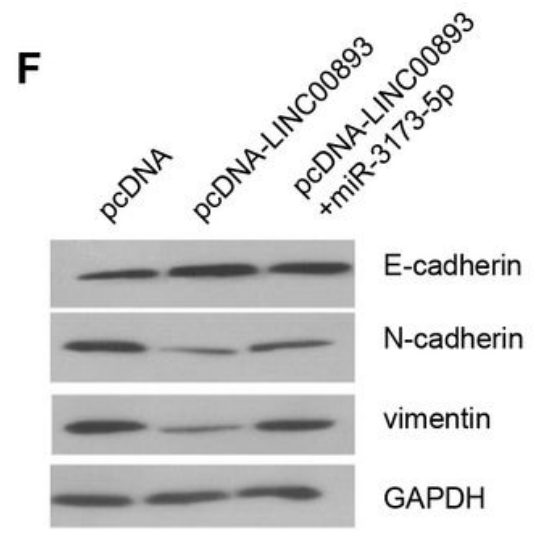

PC-3
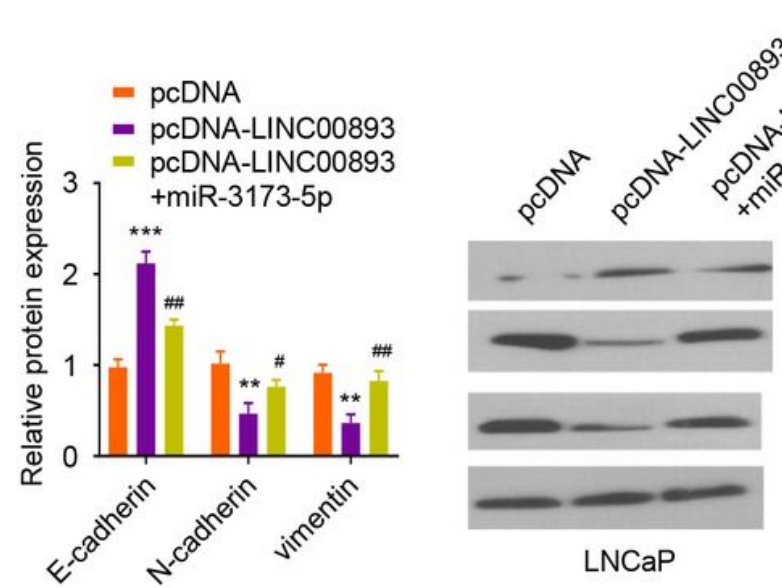

$\mathbf{E}$

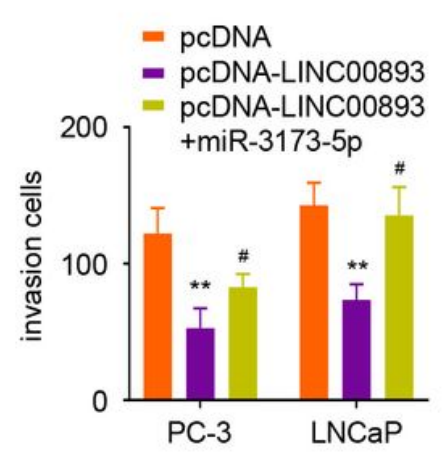

\section{Figure 4}

miR-3173-5p mediates the regulation of LINC00893 on PCa progression. PC-3 and LNCaP cells were treated as follows: pcDNA, pcDNA-LINC00893, pcDNA-LINC00893+miR-3173-5p. A and B. The proliferation ability of PCa cells under different conditions was detected by CCK-8 (A) and EdU assay (B). C. The colony formation ability of PC-3 and LNCaP cells with different indicated treatment was detected by colony formation assay. D and E. The migration and invation of PC-3 and LNCaP cells with indicated treatment was detected by transwell assay. F. EMT related proteins $\mathrm{E}$-cadherin, $\mathrm{N}$-cadherin and vimentin in cells with indicated treatment were evaluated by western blot. Three independent assays were performed 
with three technical replicates. The error bars are defined as s.d. *, P<0.05, **, P<0.01, and ***, $P<$ 0.001 .

A

WT-SOCS3 5'AGGUAUUGGGCUGGACAGGGCA-3' | | | | | hsa-miR-3173-5p 3'-UUCCUCUUUUGUCCGUCCCGU-5' MUT-SOCS3 5'-AGGUAUUGGGCUGGAAGACACA-3'
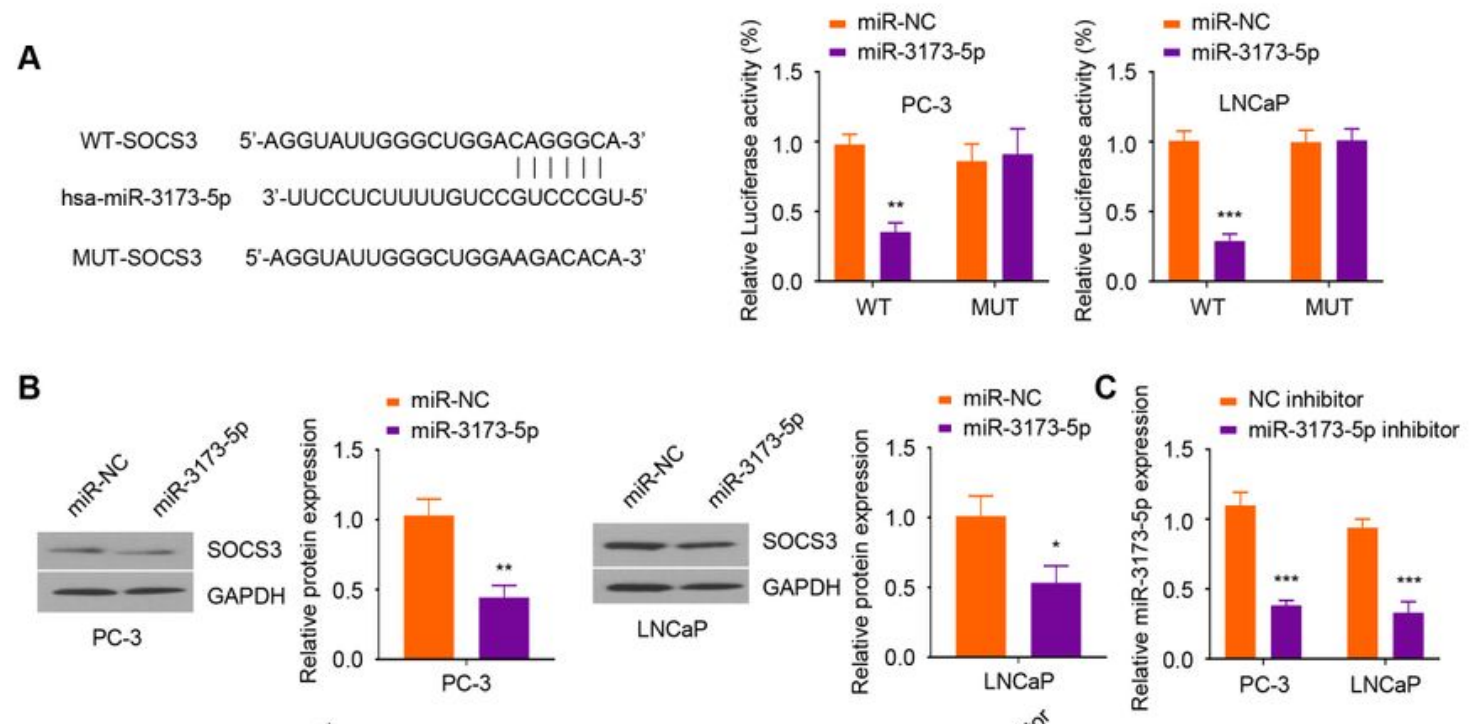

D
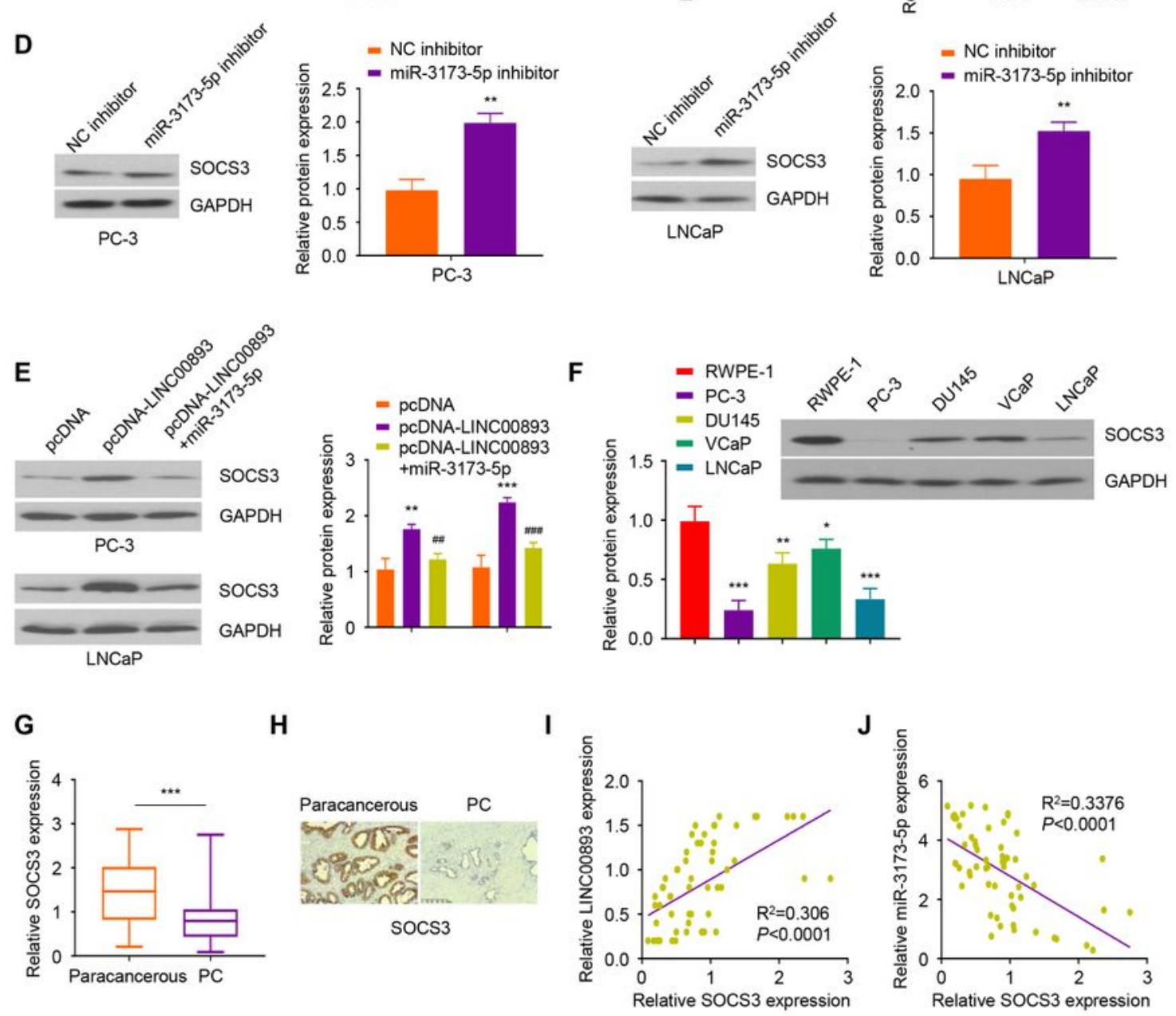

Figure 5

MiR-3173-5p binds to 3'UTR of SOCS3 mRNA and inhibits its expression. A. The binding sites of miR3173-5p on SOCS3 mRNA 3'UTR region were analyzed through Starbase database. B. SOCS3 protein expression levels was assessed by western blot after overexpression of miR-3173-5p in PC-3 and LNCaP 
cells. C. Inhibition efficiency was assessed by RT-qPCR after PC-3 and LNCaP cells were transfected with miR-3173-5p inhibitor or NC inhibitor. D and E. SOCS3 protein expression levels were assessed by western blot after cells were treated with indicated method. F. SOCS3 protein expression levels in PCa cell lines as well as human normal prostate epithelial cell line RWPE-1 were evaluated by western blot. G. SOCS3 mRNA levels in 66 pairs of PCa tissues and matched para-cancerous tissues were detected by RT-qPCR. $\mathrm{H}$. SOCS3 protein expression levels in PCa tissues and matched para-cancerous tissues were evaluated by IHC. Scale bar: $100 \mu \mathrm{M}$. I and J. The correlation between LINC00893 and SOCS3, and between MiR3173-5p and SOCS3 was analyzed by Spearman correlation coefficient analysis. Three independent assays were performed with three technical replicates. The error bars are defined as s.d. ${ }^{\star}, P<0.05, * \star, P<$ 0.01 , and $* * *, P<0.001$. 
A

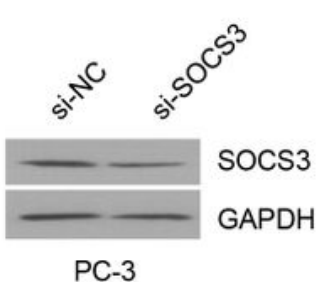

PC-3

B
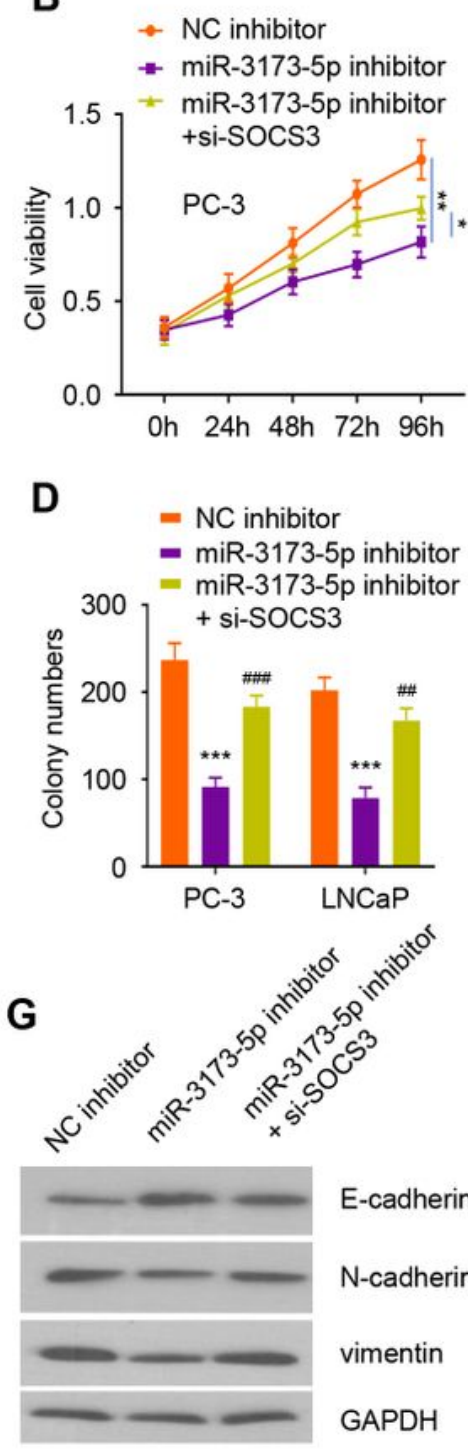

$\mathrm{E}$-cadherin
vimentin

PC-3
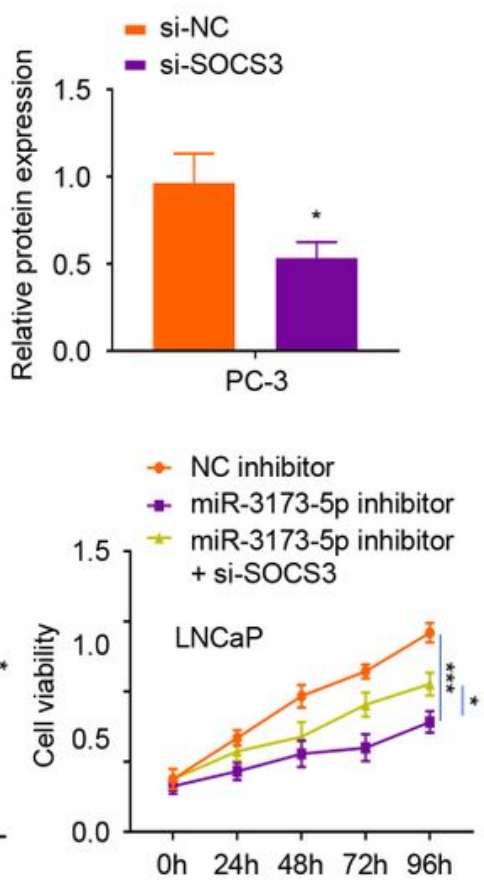

C

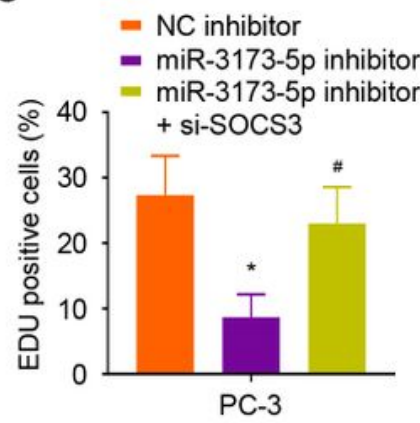

E

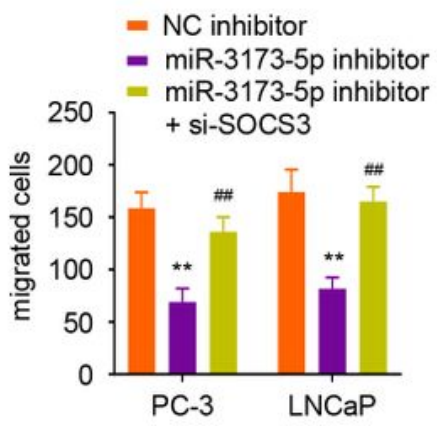

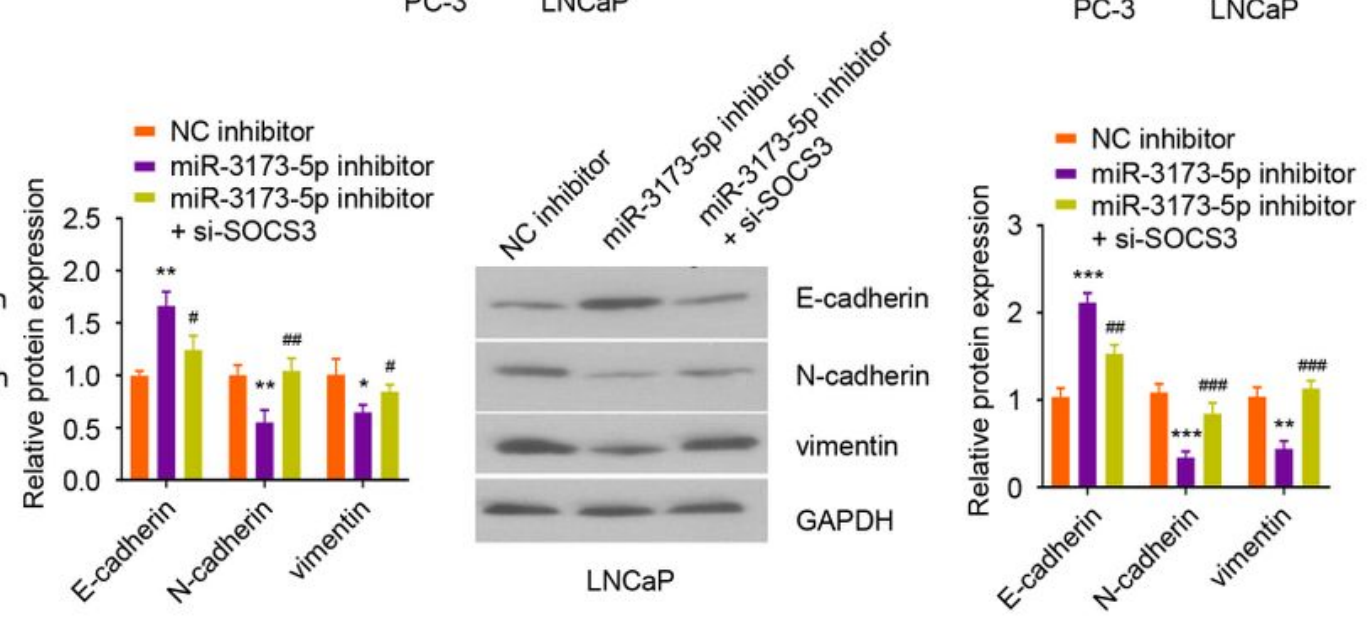
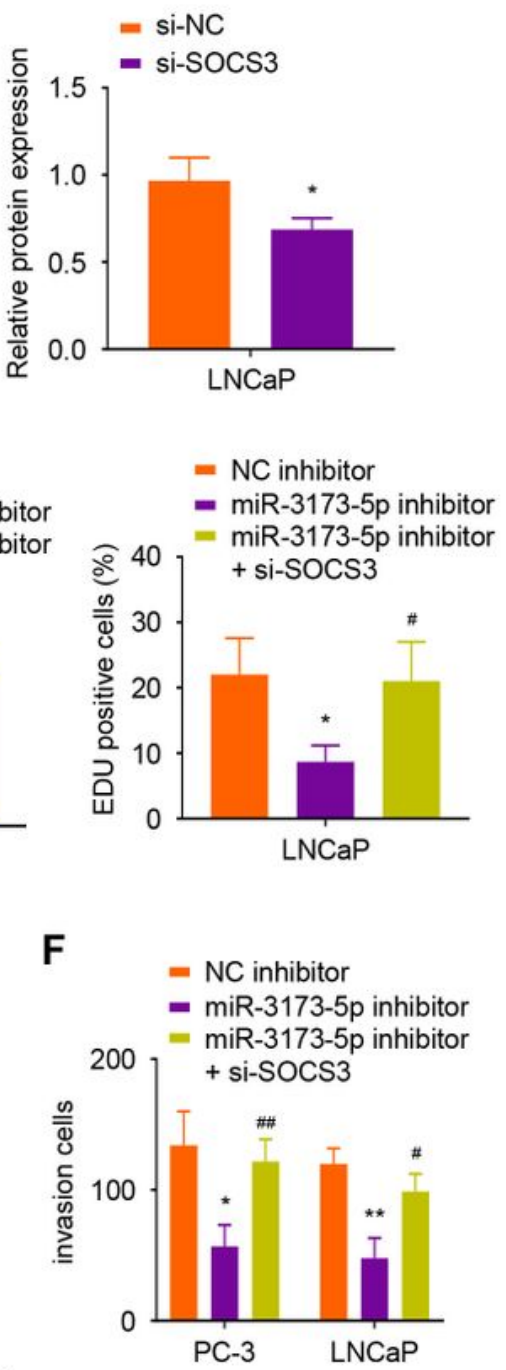

\section{Figure 6}

The enhanced malignant phenotype of PCa cells caused by miR-3173-5p knockdown was partially attenuated by SOCS3 knockdown. A. interference efficiency of SOCS3 in PC-3 and LNCaP cells transfected with si-SOCS3 or si-NC was detected by western blot. For B-G, PC-3 and LNCaP cells were transfected or co-transfected as follows: NC inhibitor $邓 m i R-3173-5 p$ inhibtor, miR-3173-5p inhibtor+siSOCS3. B and C. The proliferation ability of cells treated as indication was evaluated by CCK-8 and EdU 
assay. D. The colony formation ability of PC-3 and LNCaP cells with indicated treatment was assessed by colony formation assay. E and F. The migration (E) and invition (F) abilities of PC-3 and LNCaP cells under different conditions were evaluated by Transwell assay. G.The protein levels of EMT markers Ecadherin, $\mathrm{N}$-cadherin and vimentin in different treatment groups were detected by western blot. Three independent assays were performed with three technical replicates.. The error bars are defined as s.d. *, $P$ $<0.05, * *, P<0.01$, and $* \star *, P<0.001$.

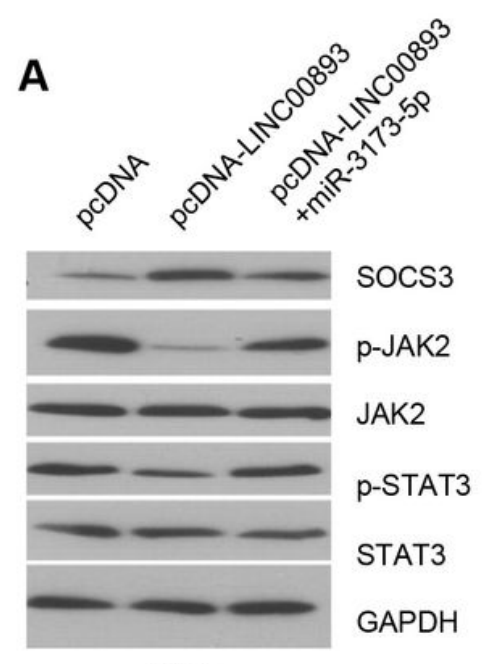

PC-3

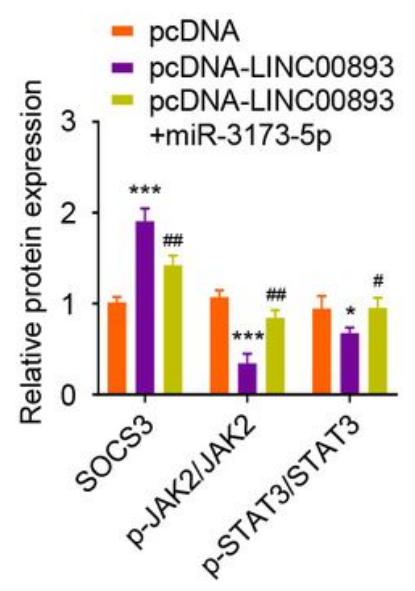

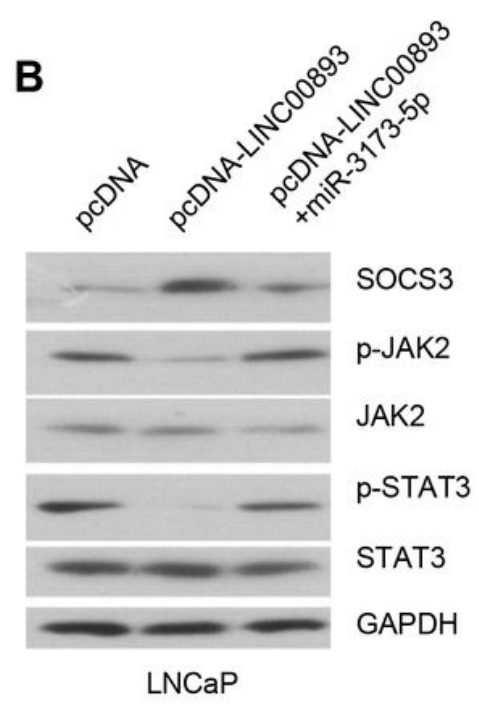

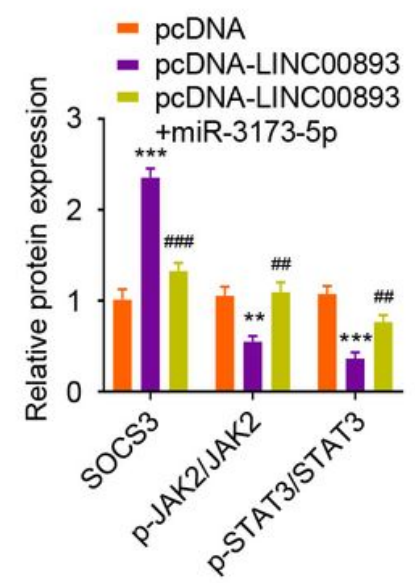

\section{Figure 7}

LINC00893 spongs miR-3173-5p and further regulates SOCS3/JAK2/STAT3 signanaling pathway. For A and $\mathrm{B}, \mathrm{PC}-3$ and LNCaP cells were treated as follows: pcDNA, pcDNA-LINC00893, pcDNALINC00893+miR-3173-5p. A and B. The levels of SOCS3/JAK2/STAT3 related proteins p-JAK2, p-STAT3, JAK2, STAT3 and SOCS3 in PC-3 (A) and LNCaP (B) cells treated as indication were assessed by western blot. Three independent assays were performed with three technical replicates. The error bars are defined as s.d. ${ }^{*}, \mathrm{P}<0.05, * \star, P<0.01$, and $* \star *, P<0.001$. 
A

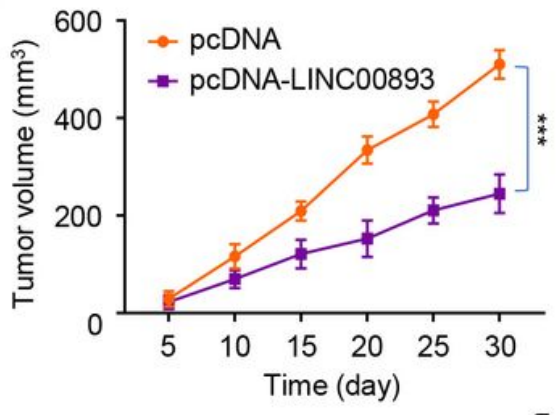

D

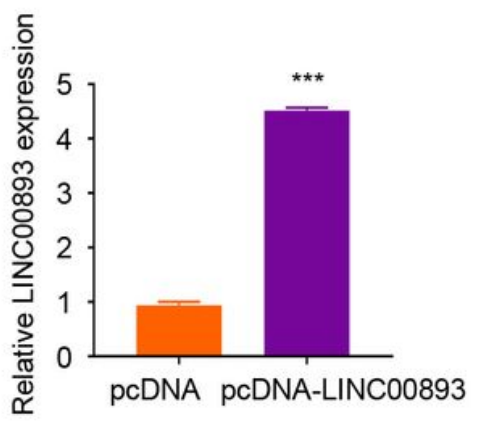

드

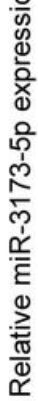

B

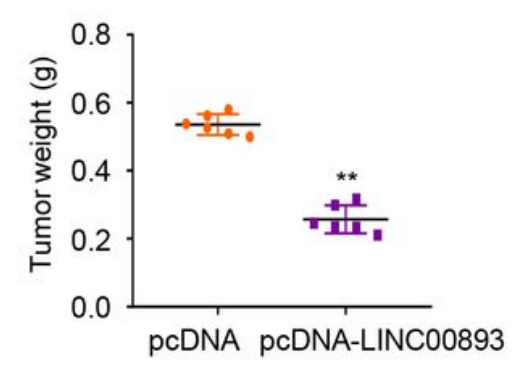

C

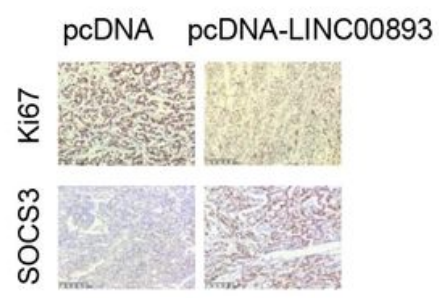

E

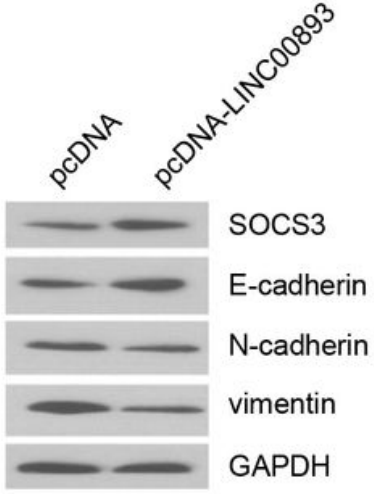

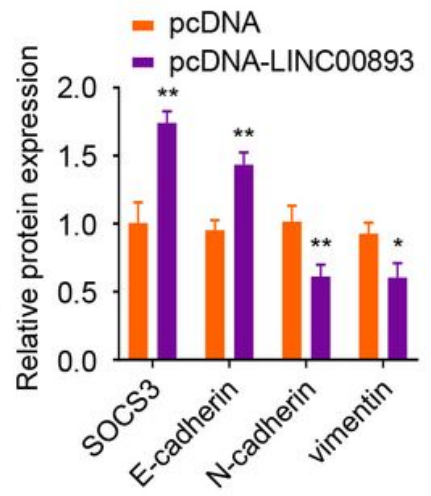

Figure 8

Overexpression of LINC00893 inhibits the proliferation of prostate cancer cells and EMT in vivo. PC-3 cells stably overexpressing LINC00893 (PC-3-LINC00893) or control cells (PC-3-vector) were inoculated into NOD/SCID mice $(n=6)$ subcutaneously at a density of $1 \times 106$. A. The volume of the tumor was detected every 5 days, and then the tumor growth curve was developed to quantify the growth rate of the tumor. B. The weight of the subcutaneous tumor was weighed in different groups. C. KI-67 and SOCS3 protein expression levels in xenograft tumor tissues was detected by IHC assay. Scale bar: $100 \mu \mathrm{M}$. D. LINC00893 and miR-3173-5p expression levels in xenograft tumor tissues was assessed by RT-qPCR. E. The protein levels of SOCS3ロE-cadherin, vimentin and $\mathrm{N}$-cadherin in $\mathrm{n}$ xenograft tumor tissues was detected by western blot. Three independent assays were performed with three technical replicates. The error bars are defined as s.d. *, $P<0.05, * *, P<0.01$, and ***, $P<0.001$. 